Department of Econometrics and Business Statistics

http://business.monash.edu/econometrics-and-business-

statistics/research/publications

\title{
On Income and Price Elasticities for Energy Demand: A Panel Data Study
}

Jiti Gao, Bin Peng and Russell Smyth

August 2020

Working Paper 28/20 


\title{
On Income and Price Elasticities for Energy Demand: A Panel Data Study ${ }^{1}$
}

\author{
Jiti Gao, Bin Peng and Russell Smyth
}

Monash University

August 15, 2020

\begin{abstract}
Obtaining reliable cross-country estimates of the income and price elasticity of energy demand requires a panel data model that can simultaneously account for endogeneity, heterogeneity, nonstationarity and cross-sectional dependence. We propose such an integrated framework and apply it to a very large dataset of 65 countries over the period 1960-2016 recently assembled by Liddle and Huntington (2020). We find that while the elasticities of income and price are non-linear, the income elasticity is generally in the range 0.6 to 0.8 and the price elasticity in the range -0.1 to -0.3 . We also find that the income elasticity has been declining since the 1990s, which broadly corresponds to increasing awareness of the negative externalities associated with burning fossil fuels associated with the Kyoto Protocol. From a policy perspective, that the income energy elasticity is less than one, and has been declining since the 1990s, bodes well for climate change mitigation because it suggests that energy intensity will fall with economic growth.

Keywords: Elasticity, Energy policy, Panel data analysis

JEL classification: C23, O13, Q11
\end{abstract}

\footnotetext{
${ }^{1}$ The first author acknowledges financial support from the Australian Research Council Discovery Grants Program under Grant Numbers: DP170104421 and DP200102769. We thank Brant Liddle who provided many helpful comments on an earlier version of this paper. The usual disclaimer applies.

Corresponding Author: Russell Smyth, Department of Economics, Monash University, Caulfield East, Victoria, Australia 3145. Email: russell.smyth@monash.edu
} 


\section{Introduction}

Energy demand has been investigated for more than half a century, employing a variety of frameworks (e.g., Brookes, 1972; Adams and Miovic, 1968; Casler, 1992; Jacobsen, 2015; Liddle et al., 2020). One of the primary interests in this literature is developing reliable estimates of the income and price elasticity of demand for energy employing panel data (e.g., Liddle et al., 2020; Liddle and Huntington, 2020 and references therein). It is important to know how price changes affect producer and consumer energy demand (e.g., Olmstead et al., 2007). Behavioural responses to price-based incentives have important implications for strategies to improve energy efficiency and reduce negative energy-related externalities (e.g., Jacobsen, 2015; Diederich and Goeschl, 2017). Given that climate mitigation strategies are often based on lowering the carbon intensity of GDP, it is equally important to know if the income elasticity is less than unity, which implies that energy intensity will fall in a business-as-usual economic growth scenario (Liddle et al., 2020).

From a methodological perspective, obtaining reliable estimates of the income and price elasticity of energy demand in a cross-country panel data setting requires a modelling framework that can simultaneously account for endogeneity, heterogeneity over time, heterogeneity across countries, nonstationarity and cross-sectional dependence.

While one, or more, of each of these five modelling issues have been addressed in panel data studies of energy demand, a limitation of the existing literature is that they have not been fully addressed within the one modelling framework. To motivate the need for a single modelling framework which simultaneously addresses endogeneity, heterogeneity over time, heterogeneity across countries, nonstationarity and cross-sectional dependence, we briefly review how each issue is present in panel data studies of energy demand, as well as outline how these issues have been addressed in the existing literature.

We begin with heterogeneity over time. Nearly all of the existing studies in the energy elasticity literature employ a parametric modelling framework that produces point, or time-invariant, estimates (see, for example, Galli, 1998; Gately and Huntington, 2002; Medlock and Soligo, 2001; van Benthem, 2015). To take account of non-linearities, these studies have mainly included the quadratic, or higher function, of GDP per capita (Galli, 1998; Medlock and Soglio, 2001) and occasionally price (van Benthem and Romani, 2009). Some of these studies employ dynamic models with a lagged dependent variable, which capture the dynamics over time to some extent. The asymmetric modeling approach to energy demand is also motivated by a desire to capture the lasting impacts of price shocks (e.g., Gately and Huntington, 2002). However, parametric models, such as these, 
are not flexible enough to fully capture non-linearities. One reason is that panels of energy data typically cover a relatively long time period, during which policies and technologies change frequently. Energy demand is also subject to episodic shocks, such as the first and second oil price shocks. One way to address this issue is to model energy demand using a non/semi-parametric approach. Semi-parametric modelling allows one to examine how the elasticities change over time. This allows for more precise estimates than the point estimates which are averages. Comparison of the time-varying estimates with the point estimates can also be informative. For instance, if the time-varying estimates do not change much over time, it might be appropriate to use accepted point estimates as a rule of thumb for forecasting (Liddle and Huntington, 2020). There are, however, very few cross-country semi-parametric panel data estimates of income and/or price elasticity of energy demand. The few examples include Karimu and Brännlund (2013), Nguyen-Van (2010), Park and Zhao (2010), Chang et al. (2016), and Liddle et al. (2020).

In addition to heterogeneity over time, it is also important to account for heterogeneity across countries. Traditionally, fixed effects have been adopted to model individual heterogeneity. Since the seminal studies by Pesaran (2006) and Bai (2009), interactive fixed effects have been incorporated in panel data models to not only capture time-varying individual heterogeneity, but also measure cross-sectional dependence. In energy demand studies, it is well-recognised that country level data exhibit cross-sectional dependence. This means that is important to account for cross-sectional dependence and time-varying individual heterogeneity using interactive fixed effects, when estimating energy elasticity using a cross-country dataset, such as the one that we use.

We next consider endogeneity. The price of energy is likely determined endogenously by the same factors that determine energy demand (see, e.g., Huntington et al., 2019). GDP per capita, as a measure of income, is also almost certainly endogenous. Microstudies of energy demand often address price endogeneity (e.g., Miller and Alberini, 2016). While some macro energy studies that have produced point estimates have explicitly addressed price endogeneity (see e.g., Burke and Abayasekara, 2018), most macro studies using panels data models just employ fixed/time effects to partially account for endogeneity (e.g., van Benthem and Romani, 2009; Nguyen-Van, 2010; Chang et al., 2016).

Finally, we consider stationarity. Many studies on energy demand assume that the regressors are stationary over time. A few studies note the importance of addressing nonstationarity (e.g., Park and Zhao, 2010; Liddle et al., 2020) and some, that have produced point estimates, have used error-correction models (see e.g. Galli, 1998). We examine the impact of income (measured by GDP per capita) and price on energy demand for 65 
countries from 1960-2016 using a dataset assembled by Liddle and Huntington (2020). Unit root tests for the variables in our study suggest that most of the time series included are nonstationary over the relevant time period. This finding is not surprising. For instance, Phillips and Moon (1999) point out that almost every macroeconomic variable in the Penn World Table is nonstationary; of which, GDP per capita is one.

To address the aforementioned issues, our main contribution in this study is to propose a (time-varying) structural equation panel data model, which simultaneously allows for endogeneity, nonstationarity, and cross-sectional dependence, and to illustrate its application to estimating income and price elasticities of energy demand using a large crosscountry dataset. We incorporate an interactive fixed effects structure into the analysis in order to capture dependence among countries and time-varying individual heterogeneity. We further adopt the approach of Casas et al. (2020), which allows for nonstationary regressors, provides a data driven method to detect the number of unobservable factors and does not limit these factors to be stationary.

It is important to note that while we use the energy elasticity literature to illustrate the application of our modelling framework, our proposed framework could be applied to several other areas of energy and environmental economics that routinely employ cross-country panel data, such as the Environmental Kuznets Curve and the relationship between income inequality and environmental degradation. While these studies typically address one or more of cross-sectional dependence, endogeneity, heterogeneity or nonstationarity, none of these studies simultaneously address all of them.

The study that is perhaps closest to ours is Liddle et al. (2020). That study also employs a subset of the Liddle and Huntington (2020) dataset for 26 countries spanning 1996-2014 to estimate income and price elasticity of energy demand using a semiparametric local linear dummy variable estimation method. We differ from that study in the following ways. First, Liddle et al. (2020) do not account for endogeneity of income or price. We explicitly address endogeneity using a traditional TSLS type estimator for both parametric and semiparametric approaches. Second, the modelling framework in Liddle et al. (2020) requires the right-hand side variables (including GDP per capita and price) to be stationary. Third, to capture heteogeneity, they use fixed effects, while we add a factor structure to capture individual and time-varying heterogeneity. Our results differ from Liddle et al. (2020) in important ways. While Liddle et al. (2020) find that the price elasticity is largely insignificant and occasionally positive, we find that both income and price elasticities are significant and vary over time. Specifically, we find that income and price elasticity of energy demand are positive and negative respectively, but income has 
stronger impacts compared to price over the time period 1960-2016.

The rest of the paper is organised as follows. Section 2 describes the details of the dataset. Section 3 presents a benchmark model and the corresponding empirical results. We present the semiparametric model and results in Section 4. Section 5 concludes.

Additional details regarding the estimation method, which is supplemental to that presented in the main text, is summarized in Appendix A.

\section{Data}

We employ a novel dataset that was assembled by Liddle and Huntington (2020), which is available at https://www.iaee.org/energyjournal/article/3508. As Liddle and Huntington (2020) note, a major weakness in most studies in this literature is the absence of long time series for energy prices for many countries, particularly non-OECD countries. The Liddle and Huntington (2020) dataset overcomes this, making it possible to employ a long panel of both OECD and non-OECD countries. Specifically, Liddle and Huntington (2020) develop a new real price index that largely stemmed from Enerdata's Global Energy and $\mathrm{CO}_{2}$ database, which begins in 1978. The initial price data are in constant US dollars. Three real indices are calculated (base year set to 2005) from the data available for three sectors: residential, industry, and transport. The final, aggregate index is a weighted average (by their share of total final energy consumption measured in tons of oil equivalent) of the three end-use indices.

The residential index is based on a weighted average of real price indices for households using PPP (including taxes) for bituminous coal, light fuel oil, electricity and natural gas. The industry index is based on a weighted average of real price indices for industry (including taxes) for bituminous coal, light fuel oil, heavy fuel oil, electricity and natural gas. To create both the residential and industry real price indices, individual sector price indices are weighted by their share in final energy consumption of the residential and industry sectors, respectively. The transport real index is based on a weighted average (again by consumption shares) of real price indices based on PPP (including taxes) of premium gasoline and diesel. Premium gasoline prices are highly correlated with unleaded gasoline prices, as are diesel prices with commercial diesel prices. The prices for premium gasoline and diesel have by far the greatest degree of coverage.

GDP per capita and total final energy consumption in toe per capita are also included in the augmented price dataset constructed by Liddle and Huntington (2020). Hereafter, we refer to the above three variables as Price, GDP and TFC for simplicity. Our objective, 
thus, is to examine how GDP and Price affect TFC. In order to account for the unbalanced nature of the original data, we use the "tidyverse" package in $\mathrm{R}$ to fill the missing values in each time series using the next entry, given that the missing values normally occur at the beginning of the time period. We further remove countries which cannot be imputed at all. Our final dataset consists of 65 countries and 57 years (1960-2016).

We take the logarithm for each variable as in Liddle et al. (2020). Given the complexity of the construction of the price variable, we normalize each time series to get mean 0 and standard deviation 1 , which ensures that every variable is unit free. For each variable, we conduct the Augmented Dickey-Fuller (ADF) test with a constant and a trending term for each time series, and report the percentage of countries exhibiting nonstationarity in Table A.1. Most time series are non-stationary. In particular, for price, more than $90 \%$ of countries fail to reject the null that the time series include a unit root. This underpins the importance of addressing stationarity within a holistic framework.

We present results for four different panels:

Dataset 1: All countries $(N=65)$ covering years 1960-2016;

Dataset 2: High income countries $(N=37)$ covering years 1960-2016;

Dataset 3: Middle income countries $(N=26)$ covering the years 1960-2016.

Dataset 4: The dataset employed in Liddle et al. (2020), which consists of a subset of 26 middle income countries covering the years 1996-2014.

For our main results, we employ the full dataset (Dataset 1). Liddle and Huntington (2020) divide their sample into middle and high-income panels, so we use Dataset 2 and 3 to compare with their results. We employ Dataset 4 primarily as a robustness check and to provide a direct comparison with the time-varying estimates in Liddle et al. (2020).

\section{The Benchmark Model}

In this section, we investigate the dataset using a constant parameter structural equation panel data model, which can be considered as a benchmark.

\subsection{The Constant Parameter Structural Equation Model}

The model is as follows. 


$$
\begin{aligned}
& y_{1 \times i t}=y_{2, i t}^{\prime} \underset{p \times 1}{\beta_{0}}+\lambda_{1, i}^{\prime} \underset{r \times 1}{f_{t}}+\varepsilon_{1, i t}, \\
& y_{2, i t}=B_{0} \underset{d \times 1}{x_{i t}}+\lambda_{2, i}^{\prime} f_{t}+\varepsilon_{2, i t}
\end{aligned}
$$

where the dimensions of the remaining variables are omitted. $x_{i t}$ includes the instrumental variables (IVs) to address endogeneity. $f_{t}$ is an $r \times 1$ unobservable factor with $r$ being unknown, $\lambda_{1, i}$ and $\lambda_{2, i}$ are unknown factor loadings and $\varepsilon_{1, i t}$ and $\varepsilon_{2, i t}$ are error terms.

Alternatively, we can write (3.1)-(3.2) in a reduced form as follows:

$$
y_{i t}=\pi_{0} x_{i t}+\Lambda_{i} f_{t}+\varepsilon_{i t}
$$

where $y_{i t}=\left(y_{1, i t}, y_{2, i t}^{\prime}\right)^{\prime}$ and $\pi_{0}$ is a matrix of unknown coefficients with dimensionality being $(p+1) \times d$. For subsequent use, we partition $\pi_{0}$ as:

$$
\pi_{0}=\left(\begin{array}{c}
\pi_{0,1} \\
1 \times d \\
\pi_{0,2} \\
p \times d
\end{array}\right) .
$$

Simple algebra shows that multiplying $(3.3)$ by $\left(1,-\beta_{0}^{\prime}\right)$ yields:

$$
\begin{aligned}
y_{1, i t}-y_{2, i t}^{\prime} \beta_{0} & =\left(1,-\beta_{0}^{\prime}\right) \pi_{0} x_{i t}+\left(1,-\beta_{0}^{\prime}\right) \Lambda_{i} f_{t}+\left(1,-\beta_{0}^{\prime}\right) \varepsilon_{i t} \\
& =\left(\pi_{0,1}-\beta_{0}^{\prime} \pi_{0,2}\right) x_{i t}+\left(1,-\beta_{0}^{\prime}\right) \Lambda_{i} f_{t}+\left(1,-\beta_{0}^{\prime}\right) \varepsilon_{i t}
\end{aligned}
$$

From (3.1) it follows:

$$
\begin{aligned}
\pi_{0,1}-\beta_{0}^{\prime} \pi_{0,2} & =0, \\
\left(1,-\beta_{0}^{\prime}\right) \Lambda_{i} & =\lambda_{1, i}^{\prime}, \\
\left(1,-\beta_{0}^{\prime}\right) \varepsilon_{i t} & =\varepsilon_{1, i t} .
\end{aligned}
$$

Once $\pi_{0}$ is recovered, we can estimate $\beta_{0}(\cdot)$ using $\pi_{0,1}-\beta_{0}^{\prime} \pi_{0,2}=0$ from (3.5) provided that $\pi_{0,2}$ has full row rank (and, thus, $d \geq p$ ).

We provide full details of the estimation procedure in Appendix A.1.

Before proceeding further, we comment on the flexibility of the above model. Consider the reduced form (3.3) for simplicity. For $r=1$, if $f_{t}=1$ ( or $\Lambda_{i}=1_{p \times 1}$ ), (3.1) reduces to a panel data structural equation model with fixed effects (or time effects). When $r=2$, (3.1) nests the case with both fixed effects and time effects as follows: 


$$
\Lambda_{i} f_{t}=\left(\begin{array}{cc}
1 & \alpha_{i, 1} \\
1 & \alpha_{i, 2} \\
1 & \alpha_{i, 3}
\end{array}\right)\left(\begin{array}{l}
\gamma_{t} \\
1
\end{array}\right)
$$

\subsection{Benchmark Results}

We present results based on (3.1)-(3.3) for three cases:

Case 1: $y_{2, i t}=\log \mathrm{GDP}_{i t}$ and $x_{i t}=\log \mathrm{GDP}_{i, t-1}$;

Case 2: $y_{2, i t}=\log$ Price $_{i t}$ and $x_{i t}=\log$ Price $_{i, t-1}$;

Case 3: $y_{2, i t}=\left(\log \mathrm{GDP}_{i t}, \log \text { Price }_{i t}\right)^{\prime}$ and $x_{i t}=\left(\log \mathrm{GDP}_{i, t-1}, \log \text { Price }_{i, t-1}\right)^{\prime}$.

We follow Liddle et al. (2020) in including the one period lag of GDP and Price, but we treat them as IVs. In addition to the coefficients of the model, we also examine the long-run GDP and Price elasticities (denoted by $E_{G}$ and $E_{P}$ hereafter). For the purpose of illustration, consider Case 3 only. Inserting (3.2) into (3.1) yields the values of $E_{G}$ and $E_{P}$, which are the first and second elements of $B_{0}^{\prime} \beta_{0}$ respectively.

In the following, we report the estimated values of $\beta_{0}, E_{G}$ and $E_{P}$. Specifically, $\beta_{0}$ includes the coefficients presented in (3.1), and so measures the impact of GDP and Price in the current period on current TFC. As explained above, $E_{G}$ and $E_{P}$ represent the elasticities. In addition, after inserting (3.2) into (3.1), one can see that $E_{G}$ and $E_{P}$ also measure the impact of GDP and Price in the previous period on current TFC.

As a point of comparison, we also report estimates from the traditional fixed effects (FE) model, by regressing $y_{1, i t} \equiv \log \mathrm{TFC}_{i t}$ on $\left(y_{2, i t}^{\prime}, x_{i t}^{\prime}\right)^{\prime}$. Thus, the model is:

$$
y_{1, i t}=\left(y_{2, i t}^{\prime}, x_{i t}^{\prime}\right) \beta_{0}+\alpha_{i}+\varepsilon_{i t}
$$

for which the long-run elasticities, $E_{G}$ and $E_{P}$, are the summation of the coefficients associated with GDP and Price respectively.

The results are presented in Tables A.2-A.5. In the FE model, in Case 2 the coefficient on Price and $E_{P}$ are positive in Tables A.2-A.5, which is inconsistent with economic theory. However, once one accounts for endogeneity, the estimates for each of the coefficients and elasticities in the structural equation model (3.1)-(3.3) have the expected sign and are significant in Tables A.2-A.5, although the magnitudes vary from dataset to dataset. Moreover, in the structural equation model, the estimates not only have the expected sign, but are significant in pretty much all cases with all datasets. 
Below, we focus on the results of the structural equation model only. First, across Tables A.2-A.5, the coefficient on GDP is always positive, while the coefficient on price is always negative, consistent with theory. With the full sample (Dataset 1), the magnitude of the elasticity for GDP is close to 1 in Table A.2. While Liddle and Huntington (2020) find that the income elasticity is generally less than unity (e.g., 0.7) for their full sample, a number of previous studies find the energy income elasticity of demand to be close to 1 (see e.g. Galli, 1998; Medlock and Soligo, 2001; van Betham, 2015). When Liddle and Huntington (2020) split the sample into high and middle-income panels, they find similar results for each panel. However, when we divide the dataset into high and middle income groups (i.e., Dataset 2 and Dataset 3), the elasticity for GDP is higher than 1 for high income countries, but lower than 1 for the middle income countries. The results for the elasticity of GDP in Dataset 4, reported in A.5, is also close to 1.

The point estimates on price vary from -0.0376 in Table A.5 to -0.2566 in Table A.3. These results are similar to those in Liddle and Huntington (2020) for their high income panel, although for their middle income panel, price was generally insignificant. For all Tables A.2-A.5, looking at the absolute values of the coefficients, $E_{G}$ and $E_{P}$, the results of the structural equation model suggest that GDP has a stronger impact on TFC than Price. This result is also consistent with the point estimates of income and price elasticities reported in studies such as Galli (1998) and Liddle and Huntington (2020).

\section{A Semiparametric Approach}

In this section, we estimate a time-varying structural equation panel data model.

\subsection{The Time-Varying Structural Equation Model}

Specifically, the model is as follows:

$$
\begin{aligned}
& y_{1, i t}=y_{2, i t}^{\prime} \underset{p \times 1}{\beta_{0}\left(\tau_{t}\right)+\lambda_{1, i t}+\varepsilon_{1, i t},} \\
& y_{2, i t}=B_{0}\left(\tau_{t}\right) \underset{d \times 1}{x_{i t}}+\lambda_{2, i t}+\varepsilon_{2, i t} .
\end{aligned}
$$

Note that both $\lambda_{1, i t}$ and $\lambda_{2, i t}$ correspond to the factor structure of the constant parameter model. The detailed form of these terms are clear from the reduced form.

We can write (4.1) and (4.2) in a reduced form as follows.

$$
y_{i t}=\pi_{0}\left(\tau_{t}\right) x_{i t}+\Lambda_{i} f_{t}+\varepsilon_{i t}
$$


where $y_{i t}=\left(y_{1, i t}, y_{2, i t}^{\prime}\right)^{\prime}$, and the other variables are defined accordingly.

We partition $\pi_{0}(\tau)$ as:

$$
\pi_{0}(\tau)=\left(\begin{array}{c}
\pi_{0,1}(\tau) \\
1 \times d \\
\pi_{0,2}(\tau) \\
p \times d
\end{array}\right)
$$

Then, similar to (3.5), the following equalities hold:

$$
\begin{aligned}
\pi_{0,1}\left(\tau_{t}\right)-\beta_{0}^{\prime}\left(\tau_{t}\right) \pi_{0,2}\left(\tau_{t}\right) & =0 \\
\left(1,-\beta_{0}^{\prime}\left(\tau_{t}\right)\right) \Lambda_{i} & =\lambda_{1, i t}, \\
\left(1,-\beta_{0}^{\prime}\left(\tau_{t}\right)\right) \varepsilon_{i t} & =\varepsilon_{1, i t} .
\end{aligned}
$$

Once $\pi_{0}(\cdot)$ is recovered, we can estimate $\beta_{0}(\cdot)$ using $(4.4)$ provided that $\pi_{0,2}(\cdot)$ has full row rank uniformly. The detailed estimation procedure is summarized in Appendix A.1.

\subsection{Constancy Test}

Before presenting the results from the semiparametric model, we first conduct a constancy test to demonstrate that a semiparametric approach is needed. In particular, we focus on (4.3), and test whether the coefficient of $\pi_{0}$ is a constant.

Formally, we state the null and alternative hypotheses below:

$$
\begin{aligned}
& \mathbb{H}_{0}: \operatorname{Pr}\left\{\pi_{0}(\cdot) \equiv \pi_{0}\right\}=1 \text { for some } \pi_{0} \in \mathbb{R}^{(p+1) \times d}, \\
& \mathbb{H}_{1}: \operatorname{Pr}\left\{\pi_{0}(\cdot) \equiv \pi_{0}\right\}<1 \text { for all } \pi_{0} \in \mathbb{R}^{(p+1) \times d}
\end{aligned}
$$

Failing to reject the null infers that a constant parameter structural equation model (3.1)(3.3) would be sufficient for examining energy demand. However, if the null is rejected, a more flexible model is needed. We summarize the technical details in Appendix A.2.

We conduct the constancy test for Case 1 to Case 3, and present the results in Table A.6. The $p$-values equal 0.000 in all tests, which strongly indicate that the constant parameter model is not flexible enough for studying energy demand.

\subsection{Results}

We consider the three cases and four datasets referred to in Section 3.2, and are interested in the values of $\beta_{0}, E_{G}$ and $E_{P}$. For Dataset 1, we report the results for all three cases. To avoid repetition, and for the sake of robustness, we report the results associated with 
Case 3 only for Datasets 2-4. Figures A.1-A.3 present the results for the income and price coefficients for each of the three cases using the data for all countries (i.e., Dataset 1), while Figures A.4-A.6 provide the results for the income and price coefficients of Case 3 using Datasets 2-4. Figures A.7-A.9 present the corresponding elasticities for Dataset 1, while Figures A.10-A.12 provide the corresponding elasticities for Datasets 2-4.

\subsubsection{Results for Dataset 1}

The coefficients and elasticities on GDP for both Case 1 and Case 3 are significant over the entire time period. While the values vary over time, in general they are less than 1 and are mostly in the range 0.6 to 0.8. In Figure A.1 we present the results for Case 1, which does not control for the price of energy. The effect of real GDP per capita on energy consumption is positive and sharply increasing from the 1990s until relatively late in the sample period. The time-varying pattern in the corresponding elasticity for Case 1, depicted in Figure A.7, is very similar. But, Figure A.1 does not control for energy prices, creating an omitted variables bias problem. Energy prices are correlated with real GDP per capita and are a determinant of energy demand. As shown Figure A.2, the coefficient on energy prices in absolute values increased markedly over a similar timeframe to which real GDP was increasing in Figure A.1. Again, the time-varying pattern in the corresponding elasticities for Case 2, depicted in Figure A.8, are very similar.

When we control for energy prices in Case 3, the time varying pattern in the income coefficient and elasticity looks very different. The mildly non-linear inverted U-shaped pattern in the income coefficients and elasticities in Figure A.3 and Figure A.9 is consistent with the dematerialization process emphasised by authors such as Brookes (1972) in which energy intensity initially increases as low-income countries increase their industrial bases and then declines over time with a sectoral shift from energy-intensive heavy industry to light industry and then finally to the less energy-intensive commercial sector. Studies such as Medlock and Soligo (2001) document this phenomenon using the quadratic of GDP in a parametric specification, as do Chang et al. (2016) in a nonparametric framework for specific countries, such as China and South Korea.

The finding that the income coefficient and elasticities in Figure A.3 and Figure A.9 have been declining since the 1990s is consistent with the time-varying estimates for GDP in Chang et al. (2016) and Liddle et al., 2020. Most countries have experienced a decrease in energy intensity, defined as the ratio of energy consumption to real GDP, over the last two decades. Since 1990, global energy intensity has declined at an average rate of 1.2 per cent per year, while for lower-middle income countries this figure has been higher 
at 1.8 per cent per year (see e.g. Chen et al., 2019). Hence, the decline in the income coefficient and elasticities in Figure A.3 and Figure A.9 since the 1990s is consistent with the increase in autonomous energy efficiency over the same period.

What explains the decreasing income coefficient and elasticity in Figure A.3 and Figure A.9 since the 1990s? One important event was the Kyoto Protocol, which was signed in 1997. As Chang et al. (2016) note, the Kyoto Protocol marked a major milestone in increasing awareness of the negative externalities of consuming fossil fuels. It resulted in many high-and-middle income countries reducing their income elasticity with the goal of lowering pollution, consistent with being on the downward sloping section of the Environmental Kuznets Curve. More generally, it represented "the culmination of shifting attitudes [on climate change] up to that time" (Chang et al., 2016, p. 240).

The decline in the income coefficient in Figure A.3 accelerates from the mid-2000s. This coincides with signatories to the Kyoto Protocol being required to implement efficiency measures by 2005 and growing public awareness of the dangers posed by climate change (for a detailed review of changing attitudes on climate change from opinion poll data see Capstick et al., 2015). It also reflects the effect of the Global Financial Crisis, which reduced economic growth and energy intensity (Andreoni, 2020).

Consistent with the findings in Section 3.2, the coefficient and elasticity on Price is negative and significant in Case 2 and 3 over almost the entire time period with the magnitudes in the range -0.1 to -0.3 . In both Figure A.3 and Figure A.9 the pattern is somewhat wave shaped. For the period from the mid-1960s to the mid-1980s there is a U-shape, consistent with Fouquet (2014) who found that price elasticities follow a u-shape pattern as the economy develops. Since the 1990s, energy demand has become more price elastic. This likely reflects the effect of the Kyoto Protocol and substitution towards renewable energy sources and the economic downturn in the Global Financial Crisis. Consistent with this interpretation, there are also short periods in which energy demand becomes more price sensitive following the first and second oil price shock. This result is also consistent with the meta-analysis by Labandeira et al. (2017) which found that energy demand becomes less sensitive following an energy crisis or recession.

In Figure A.3, the magnitude on the price coefficient is lower in absolute terms than the coefficient on GDP over the entire period. The same is true for the corresponding elasticities - $E_{G}$ and $E_{P}$ - in Figure A.9, which reinforces the conclusion from the point estimates that GDP has stronger impacts on TFC compared to Price.

It is worth noting that using the semiparametric approach, two unobservable factors are identified (i.e., $r=2$ ) for all Cases 1-3. However, the parametric approach identifies 
only one unobservable factor (see Table A.2). This results further reinforces the need to examine the elasticity of energy demand in a semiparametric setting, given that more information can be retrieved. Finally, we emphasize that as shown in Figures A.1-A.3 and A.7-A.9, our approach is not sensitive to the choices of bandwidth.

\subsubsection{Results for Datasets 2-4}

In Figures A4 and A10 we present the Case 3 coefficient and elasticity results for the high-income panel (Dataset 2). The coefficient on GDP per capita is positive and significant and generally in the range 0.8 to 1 , although it peaked in the mid-1970s at 1.2 . The coefficient on GDP per capita is highly non-linear. It first exhibits a U-shaped pattern followed by an inverted U-shaped pattern. The latter, consistent with the dematerialization process, is much more pronounced in the high-income sample from the mid-1960s through to the end of the sample period. The wave-like pattern in GDP per capita is consistent with findings from parametric studies of energy demand in high-income countries. Galli (1998), Medlock and Soligo (2001) and van Bethem and Romani (2009) estimated a Ushaped relationship for GDP per capita at low income intervals, but an inverted U-shape relationship at higher income ranges. The coefficient on price is negative and significant with magnitudes in the range -0.1 to -0.2 throughout. The corresponding elasticities in Figure A10 exhibit a similar time-varying pattern to the coefficients.

In Figures A5 and A11 we present the Case 3 coefficient and elasticity results for the middle-income panel (Dataset 3). The coefficient on GDP per capita is positive and significant and the magnitude is around 0.7 to 0.8 for most of the period. A difference between Figures A4 and A5, is that in the latter the non-linear inverted U-shaped pattern in GDP per capita occurs later in the sample period. Specifically, the hump shape in GDP begins in the mid-1990s and extends to the end of the sample period, with the peak at 1 around the time of the Global Financial Crisis, which is consistent with these countries being both later to industrialize and then gradually transition to service economies.

The coefficient on price is negative and significant for most of the period with magnitudes being in the range -0.1 to -0.2 , although the confidence intervals cut the horizontal axis at the beginning and end of the sample period. Recall that Liddle and Huntington (2020) found the point estimates for price to be mostly insignificant for the middle-income panel. The time-varying estimates suggest that the findings for the point estimates could reflect the situation at the beginning and end of the sample period. The time-varying pattern in the corresponding elasticities in Figure A11 are similar to the coefficients.

Similar to the findings of the point estimates in Section 3.2, the elasticity for GDP 
of the high income countries is higher than that of the middle income countries. Meanwhile, the elasticity for Price of the high income countries is roughly the same as that of the middle income countries. Moreover, in both figures, the absolute magnitude of the elasticity for Price is much less than the elasticity for GDP across the entire period.

In Figures A.6 and A.12, we provide the results using the Liddle et al. (2020) dataset. In Figure A. 6 the coefficient on GDP per capita is around 0.9 throughout the period. The coefficient on price is in the range -0.1 to -0.2 , although it is not statistically different from zero from around the time of the Global Financial Crisis onwards. In Figure A.12 we present the corresponding elasticities. The time-varying pattern in the elasticities are similar to the coefficients, although the confidence interval for price elasticity cuts the horizontal axis earlier than the price coefficient in Figure A.6.

The coefficient plots associated with GDP for the two middle income samples (Datasets 3 and 4) are generally similar to those presented in Figure 3.b in Liddle et al. (2020). In that study, the coefficient on income was generally in the range 0.6 to 0.8 between 1996 and 2014. However, comparing the results for price from Datasets 3 and 4 with those in Figure 3.b in Liddle et al. (2020), we find more evidence that the coefficient on price is negative and significant for extended periods. Possible explanations for the different results is that in Liddle et al. (2020) the data have been detrended nonparametrically and that Liddle et al. (2020) do not account for endogeneity of price.

\section{Conclusion}

Estimating energy elasticities with panel data models present five main modelling challenges: ie. addressing endogeneity, heterogeneity over time, heterogeneity across units, nonstationarity and cross-sectional dependence. While there is a large literature estimating energy elasticities with panel data, these five issues have not been fully addressed in an integrated framework. Our contribution has been to propose such a framework and apply it to a very large panel recently assembled by Liddle and Huntington (2020) that includes data on energy prices as well as GDP and spans 56 years. Analysing such a large dataset which extends back to 1960 means that not only do we employ a model that simultaneously addresses a range of issues that has plagued this literature, but we present time-varying estimates for energy income and price elasticity that are, by far, for the longest time period and widest cross-section of countries available.

We find that the elasticities of income and price are time-varying. For Dataset 1, in Case 3 in which we include income and price in the one specification, the income 
elasticity is generally in the range 0.6 to 0.8 , while the price elasticity in the range -0.1 to -0.3 , although the income elasticity has been decreasing since the 1990s. The results for the income elasticity are generally consistent with the notion of dematerialization in the energy literature. The decline in the income elasticity since the 1990s is consistent with the effect of the Kyoto Protocol reducing energy intensity.

From a policy perspective, that the income energy elasticity is less than one, and has been declining since the 1990s, bodes well for climate change mitigation because it suggests that energy intensity will fall with economic growth. More generally, consistent with the conclusion in Chang et al. (2016), our findings point to transnational institutions, such as the Kyoto Protocol and the Paris Agreement, having an important role in reducing energy intensity, both as commitment devices for the signatories and as barometers of changing public attitudes toward fossil fuels and climate change.

\section{References}

Adams, F. G. and Miovic, P. (1968), 'On relative fuel efficiency and the output elasticity of energy consumption in western europe', Journal of Industrial Economics 17(1), 41-56.

Andreoni, V. (2020), 'The energy metabolism of countries: Energy efficiency and use in the period that followed the global financial crisis', Energy Policy 139, 111304.

Bai, J. (2009), 'Panel data models with interactive fixed effects', Econometrica 77(4), 12291279.

Brookes, L. G. (1972), 'More on the output elasticity of energy consumption', Journal of Industrial Economics 21(1), 83-92.

Burke, P. J. and Abayasekara, A. (2018), 'The price elasticity of electricity demand in the united states: A three-dimensional analysis', Energy Journal 39(2), 123-145.

Capstick, S., Whitmarsh, L., Poortinga, W., Pidgeon, N. and Upham, P. (2015), 'International trends in public perceptions of climate change over the past quarter century', WIREs Climate Change 6(1), 35-61.

Casas, I., Gao, J., Peng, B. and Xie, S. (2020), Time-varying income elasticities of healthcare expenditure for the OECD and Eurozone. https://ssrn.com/abstract=3262326.

Casler, S. D. (1992), 'Energy demand and the composition of output growth', Journal of Environmental Economics and Management 22(2), 114 - 133. 
Chang, Y., Choi, Y., Kim, C. S., Miller, J. I. and Park, J. Y. (2016), 'Disentangling temporal patterns in elasticities: A functional coefficient panel analysis of electricity demand', Energy Economics 60, 232 - 243.

Chen, Z., Huang, W. and Zheng, X. (2019), 'The decline in energy intensity: Does financial development matter ?', Energy Policy 134, 110945.

Diederich, J. and Goeschl, T. (2017), 'To mitigate or not to mitigate: The price elasticity of proenvironmental behavior', Journal of Environmental Economics and Management 84(C), 209222.

Fouquet, R. (2014), 'Long-run demand for energy services: Income and price elasticities over two hundred years', Review of Environmental Economics and Policy 8, 186-207.

Galli, R. (1998), 'The relationship between energy intensity and income levels: Forecasting long term energy demand in asian emerging countries', The Energy Journal 19(4), 85-105.

Gately, D. and Huntington, H. (2002), 'The asymmetric effects of changes in price and income on energy and oil demand', The Energy Journal 23(1), 19-55.

Huntington, H. G., Barrios, J. J. and Arora, V. (2019), 'Review of key international demand elasticities for major industrializing economies', Energy Policy 133, 110878.

Jacobsen, G. D. (2015), 'Do energy prices influence investment in energy efficiency? Evidence from energy star appliances', Journal of Environmental Economics and Management 74, 94 $-106$.

Karimu, A. and Brännlund, R. (2013), 'Functional form and aggregate energy demand elasticities: A nonparametric panel approach for 17 OECD countries', Energy Economics 36, 19-27.

Labandeira, X., Labeaga, J. M. and López-Otero, X. (2017), 'A meta-analysis on the price elasticity of energy demand', Energy Policy 102, 549 - 568 .

Liddle, B. and Huntington, H. (2020), 'Revisiting the income elasticity of energy consumption: A heterogeneous, common factor, dynamic oecd \& non-oecd country panel analysis', Energy Journal 41(3), 207-229.

Liddle, B., Smyth, R. and Zhang, X. (2020), 'Time-varying income and price elasticities for energy demand: Evidence from a middle-income panel', Energy Economics 86, 1-10.

Medlock, K. B. and Soligo, R. (2001), 'Economic development and end-use energy demand', The Energy Journal 22(2), 77-105. 
Miller, M. and Alberini, A. (2016), 'Sensitivity of price elasticity of demand to aggregation, unobserved heterogeneity, price trends, and price endogeneity: Evidence from U.S. data', Energy Policy 97, 235 - 249.

Nguyen-Van, P. (2010), 'Energy consumption and income: A semiparametric panel data analysis', Energy Economics 32(3), 557 - 563.

Olmstead, S. M., Michael Hanemann, W. and Stavins, R. N. (2007), 'Water demand under alternative price structures', Journal of Environmental Economics and Management 54(2), 181 $-198$.

Park, S. Y. and Zhao, G. (2010), 'An estimation of u.s. gasoline demand: A smooth time-varying cointegration approach', Energy Economics 32(1), 110 - 120.

Pesaran, M. H. (2006), 'Estimation and inference in large heterogeneous panels with a multifactor error structure', Econometrica 74(4), 967-1012.

Phillips, P. C. B. and Moon, H. R. (1999), 'Linear regression limit theory for nonstationary panel data', Econometrica 67(5), 1057-1111.

Su, L. and Wang, X. (2017), 'On time-varying factor models: Estimation and testing', Journal of Econometrics 198(1), 84-101.

van Benthem, A. A. (2015), 'Energy leapfrogging', Journal of the Association of Environmental and Resource Economists 2(1), 93-132.

van Benthem, A. A. and Romani, M. (2009), 'Fuelling growth: What drives energy demand in developing countries ?', Energy Journal 30(3), 91-114. 


\section{Appendix A}

The Appendix presents materials supplemental to the main text. First, in Appendix A.1, we describe the estimation procedure for the time-varying structural equation panel data model (4.1)-(4.3). The estimation procedure for the constant parameter model (3.1)-(3.3) can be considered as a much simplified version, so is omitted. Section A.2 provides the details of the constancy test. The tables and figures are summarized in Section A.3.

\section{A.1 The Estimation Procedure for (4.1)-(4.3)}

We focus on the estimation of $\pi_{0}(\cdot)$ of $(4.3)$. Write (4.3) in matrix form as follows.

$$
Y_{i}=X_{i, \pi_{0}}+\Gamma_{i, F}+\mathcal{E}_{i}
$$

where $Y_{i}=\left(y_{i 1}, \ldots, y_{i T}\right)^{\prime}, X_{i, \pi_{0}}=\left(\pi_{0}\left(\tau_{1}\right) x_{i 1}, \ldots, \pi_{0}\left(\tau_{T}\right) x_{i T}\right)^{\prime}, \Gamma_{i, F}=\left(\Lambda_{i} f_{1}, \ldots, \Lambda_{i} f_{T}\right)^{\prime}$ and $\mathcal{E}_{i}=\left(\varepsilon_{i 1}, \ldots, \varepsilon_{i T}\right)^{\prime}$.

We adopt the approach of Casas et al. (2020). First, define the following objective function.

$$
Q(\pi, \mathcal{F})=\operatorname{tr}\left\{\sum_{i=1}^{N}\left(Y_{i}-X_{i} \pi^{\prime}\right)^{\prime} \mathrm{K}_{h, \tau} M_{\mathcal{F}} \mathrm{K}_{h, \tau}\left(Y_{i}-X_{i} \pi^{\prime}\right)\right\},
$$

where

1. $\pi$ is $(p+1) \times d, \quad X_{i}=\left(x_{i 1}, \ldots, x_{i T}\right)^{\prime}, \operatorname{tr}\{\cdot\}$ stands for the trace operation;

2. $\mathrm{K}_{h, \tau}=\operatorname{diag}\left\{\sqrt{K_{h}\left(\tau_{1}-\tau\right)}, \ldots, \sqrt{K_{h}\left(\tau_{T}-\tau\right)}\right\}, K_{h}(\cdot)=K(\cdot / h) / h$, and $K(\cdot)$ and $h$ are the kernel function and bandwidth respectively;

3. $\mathcal{F}=\left(F_{1}, \cdots, F_{T}\right)^{\prime}$, and $M_{\mathcal{F}}=I_{T}-\mathcal{F}\left(\mathcal{F}^{\prime} \mathcal{F}\right)^{-1} \mathcal{F}^{\prime}$ provided $\mathcal{F}$ has full column rank.

The detailed steps are as follows.

Step 1: For $\forall \tau \in[0,1]$, we estimate $\pi_{0}(\tau)$ and $F=\left(f_{1}, \ldots, f_{T}\right)^{\prime}$ by

$$
(\widehat{\pi}(\tau), \widehat{F})=\operatorname{argmin} Q(\pi, \mathcal{F}) \text { subject to } \frac{1}{T} \mathcal{F}^{\prime} \mathcal{F}=I_{J}, J \geq r,
$$

where $J$ is a user chosen large constant and $r$ is defined as in Section 4.1.

Step 2: Let $\widehat{\eta}_{j}$ be the $j^{\text {th }}$ largest eigenvalue of the estimated sample covariance matrix

$$
\widehat{\Sigma}(\tau)=\frac{1}{N} \sum_{i=1}^{N} \mathrm{~K}_{h, \tau}\left(Y_{i}-X_{i} \widehat{\pi}^{\prime}(\tau)\right)\left(Y_{i}-X_{i} \widehat{\pi}^{\prime}(\tau)\right)^{\prime} \mathrm{K}_{h, \tau} .
$$


Define a mock eigenvalue $\widehat{\eta}_{0}:=\left\|\frac{1}{N} \sum_{i=1}^{N}\left(Y_{i}-X_{i} \widehat{\pi}^{\prime}(\tau)\right)^{\prime} \mathrm{K}_{h, \tau}^{2}\left(Y_{i}-X_{i} \widehat{\pi}^{\prime}(\tau)\right)\right\|_{2}$, where $\|\cdot\|_{2}$ stands for the spectral norm. We estimate $r$ by

$$
\widehat{r}=\sup _{\tau \in[0,1]} \underset{1 \leq \ell \leq J}{\operatorname{argmin}}\left\{\frac{\widehat{\eta}_{\ell+1}}{\widehat{\eta}_{\ell}} \cdot \mathbb{I}\left(\frac{\widehat{\eta}_{\ell}}{\widehat{\eta}_{0}} \geq \epsilon_{N}\right)+\mathbb{I}\left(\frac{\widehat{\eta}_{\ell}}{\widehat{\eta}_{0}}<\epsilon_{N}\right)\right\},
$$

where $\epsilon_{N}=\left\{\ln \left(\max \left\{\widehat{\eta}_{0}, N\right\}\right)\right\}^{-1}$.

Step 3: Update the estimates of $\pi_{0}(\tau)$ and $F$ by

$$
(\widetilde{\pi}(\tau), \widetilde{F})=\operatorname{argmin} Q(\pi, \mathcal{F}) \text { subject to } \frac{1}{T} \mathcal{F}^{\prime} \mathcal{F}=I_{J}, J \geq \widehat{r} \text {. }
$$

$\widetilde{\pi}(\tau)$ and $\widetilde{F}$ are the final estimates of $\pi_{0}(\tau)$ and $F$.

Finally, we comment on the choice of kernel function and the bandwidth. We follow Su and Wang (2017) and adopt the boundary adjusted kernel as follows.

$$
K\left(\left(\tau_{t}-\delta\right) / h\right)= \begin{cases}\mathcal{K}\left(\left(\tau_{t}-\delta\right) / h\right) / \int_{-\delta / h}^{1} \mathcal{K}(w) d w, & \delta \in[0, h) \\ \mathcal{K}\left(\left(\tau_{t}-\delta\right) / h\right), & \delta \in[h, 1-h] \\ \mathcal{K}\left(\left(\tau_{t}-\delta\right) / h\right) / \int_{-1}^{(1-\delta) / h} \mathcal{K}(w) d w, & \delta \in(1-h, 1]\end{cases}
$$

for $t=1, \ldots, T$, where $\mathcal{K}(w)$ is the Epanechnikov kernel.

For all estimates conducted in this paper, we select $h=0.2679$, which provides around $2 \cdot\lfloor T h\rfloor \approx 30$ time periods along the time dimension. To examine the sensitivity of bandwidth, we further consider $h_{L}=0.2411$ and $h_{R}=0.2947$, which varies the magnitude of the bandwidth by $\pm 10 \%$.

\section{A.2 The Constancy Test}

This section provides the details of the constancy test.

Step 1: Under the null, we estimate $\pi_{0}$ and $F$ by

$$
(\widehat{\pi}, \widehat{F})=\operatorname{argmin} Q^{*}(\pi, \mathcal{F}) \text { subject to } \frac{1}{T} \mathcal{F}^{\prime} \mathcal{F}=I_{J}, J \geq r
$$

where $J$ is a user chosen large constant, and

$$
Q^{*}(\pi, \mathcal{F})=\operatorname{tr}\left\{\sum_{i=1}^{N}\left(Y_{i}-X_{i} \pi^{\prime}\right)^{\prime} M_{\mathcal{F}}\left(Y_{i}-X_{i} \pi^{\prime}\right)\right\} .
$$

Step 2: Let $\widehat{\eta}_{j}^{*}$ be the $j^{\text {th }}$ largest eigenvalue of the estimated sample covariance matrix 


$$
\widehat{\Sigma}^{*}=\frac{1}{N} \sum_{i=1}^{N}\left(Y_{i}-X_{i} \widehat{\pi}^{\prime}\right)\left(Y_{i}-X_{i} \widehat{\pi}^{\prime}\right)^{\prime}
$$

Define a mock eigenvalue $\widehat{\eta}_{0}^{*}:=\left\|\frac{1}{N} \sum_{i=1}^{N}\left(Y_{i}-X_{i} \widehat{\pi}^{\prime}\right)^{\prime}\left(Y_{i}-X_{i} \widehat{\pi}^{\prime}\right)\right\|_{2}$. We estimate $r$ by

$$
\widehat{r}=\underset{1 \leq \ell \leq J}{\operatorname{argmin}}\left\{\frac{\widehat{\eta}_{\ell+1}^{*}}{\widehat{\eta}_{\ell}^{*}} \cdot \mathbb{I}\left(\frac{\widehat{\eta}_{\ell}^{*}}{\widehat{\eta}_{0}^{*}} \geq \epsilon_{N}\right)+\mathbb{I}\left(\frac{\widehat{\eta}_{\ell}^{*}}{\widehat{\eta}_{0}^{*}}<\epsilon_{N}\right)\right\}
$$

where $\epsilon_{N}=\left\{\ln \left(\max \left\{\widehat{\eta}_{0}^{*}, N\right\}\right)\right\}^{-1}$.

Step 3: Update the estimates of $\pi_{0}$ and $F$ by

$$
(\widetilde{\pi}, \widetilde{F})=\operatorname{argmin} Q(\pi, \mathcal{F}) \text { subject to } \frac{1}{T} \mathcal{F}^{\prime} \mathcal{F}=I_{J}, J \geq \widehat{r} \text {. }
$$

Let further $\widetilde{u}_{i t}=y_{i t}-\tilde{\pi} x_{i t}-\widetilde{\Lambda}_{i} \widetilde{f}_{t}$ and $\widetilde{\Lambda}_{i}=\frac{1}{T}\left(Y_{i}-X_{i} \widetilde{\pi}^{\prime}\right)^{\prime} \widetilde{F}$, where $\widetilde{F}=\left(\widetilde{f}_{1}, \ldots, \widetilde{f}_{T}\right)^{\prime}$. Calculate the statistic:

$$
L_{N T}=\frac{1}{N T \sqrt{h^{*}}} \sum_{i, j} \sum_{t \neq s} 1_{3 \times 1}^{\prime} \widetilde{u}_{i t} \widetilde{u}_{j s} 1_{3 \times 1} K\left(\frac{\tau_{t}-\tau_{s}}{h^{*}}\right)
$$

where $h^{*}$ is a bandwidth, and $\sum_{i, j}$ and $\sum_{t \neq s} \operatorname{read} \sum_{i=1}^{N} \sum_{j=1}^{N}$ and $\sum_{t=1}^{T} \sum_{t=1, t \neq s}^{T}$, respectively. By Corollary 2.1 of Casas et al. (2020), $L_{N T} \rightarrow_{D} N\left(0, \sigma_{\ell}^{2}\right)$, where $\sigma_{\ell}^{2}=$ $2 \delta_{u}^{4} \int K^{2}(w) d w$, in which $\delta_{u}^{2}$ can be estimated by $B_{N T}=\frac{1}{N T} \sum_{t=1}^{T}\left(\sum_{i=1}^{N} 1_{3 \times 1}^{\prime} \widetilde{u}_{i t}\right)^{2}$ consistently. 


\section{A.3 Tables and Figures}

Table A.1: Percentage of Countries Exhibiting Nonstationarity for Each Variable

\begin{tabular}{lr}
\hline \hline Variable & Percentage \\
TFC & $64.62 \%$ \\
GDP & $72.31 \%$ \\
Price & $92.31 \%$ \\
\hline \hline
\end{tabular}


Table A.2: Results of the Constant Parameter Models from All Countries (i.e., Dataset 1). The numbers in parentheses are the 95\% confidence interval.

\begin{tabular}{|c|c|c|c|c|c|c|c|}
\hline \multirow{6}{*}{ Coefficient $\beta_{0}$} & \multirow{4}{*}{ GDP } & \multicolumn{3}{|c|}{ The Fixed Effects Model (3.6) } & \multicolumn{3}{|c|}{ The Structural Equation Model (3.1)-(3.3) } \\
\hline & & \multirow{2}{*}{$\begin{array}{l}\text { Case } 1 \\
0.0601\end{array}$} & Case 2 & \multirow{2}{*}{$\begin{array}{l}\text { Case } 3 \\
0.1090\end{array}$} & \multirow{2}{*}{$\begin{array}{l}\text { Case } 1 \\
0.9920\end{array}$} & \multirow[t]{2}{*}{ Case 2} & \multirow{2}{*}{$\frac{\text { Case } 3}{0.9946}$} \\
\hline & & & & & & & \\
\hline & & $(-0.1127,0.2961)$ & & $(-0.0797,0.2977)$ & $(0.9600,1.0331)$ & & $(0.9696,1.0266)$ \\
\hline & Price & & 0.1707 & -0.0373 & & -0.1945 & -0.0654 \\
\hline & & & $(0.0866,0.2777)$ & $(-0.0974,0.0262)$ & & $(-0.2162,-0.1691)$ & $(-0.0889,-0.0475)$ \\
\hline \multirow{9}{*}{$\begin{array}{l}\text { No. of Factors } \\
\text { Elasticity }\end{array}$} & $\operatorname{GDP}(-1)$ & 0.5775 & & 0.6443 & & & \\
\hline & & $(0.3314,0.7448)$ & & $(0.4699,0.8348)$ & & & \\
\hline & Price $(-1)$ & & -0.0254 & -0.1946 & & & \\
\hline & & & $(-0.1302,0.0688)$ & $(-0.2634,-0.1284)$ & & & \\
\hline & & & & & 1 & 1 & 1 \\
\hline & $E_{G}$ & 0.6376 & & 0.7533 & 0.9566 & & 0.9542 \\
\hline & & $(0.6055,0.6670)$ & & $(0.7257,0.7837)$ & $(0.9360,0.9846)$ & & $(0.9416,0.9803)$ \\
\hline & $E_{P}$ & & 0.1453 & -0.2319 & & -0.1723 & -0.0574 \\
\hline & & & $(0.1114,0.1777)$ & $(-0.2596,-0.2071)$ & & $(-0.1923,-0.1508)$ & $(-0.0698,-0.0436)$ \\
\hline
\end{tabular}


Table A.3: Results of the Constant Parameter Models from the High Income Countries (i.e., Dataset 2). The numbers in parentheses are the $95 \%$ confidence interval.

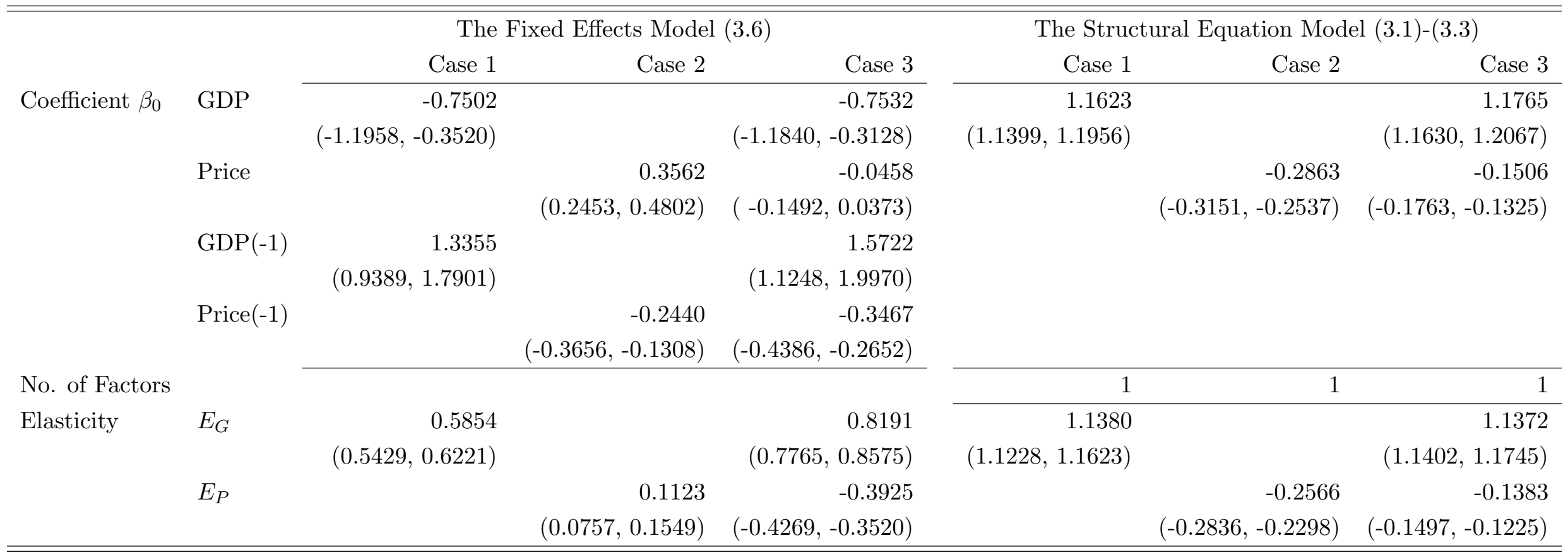


Table A.4: Results of the Constant Parameter Models from the Middle Income Countries (i.e., Dataset 3). The numbers in parentheses are the $95 \%$ confidence interval.

\begin{tabular}{|c|c|c|c|c|c|c|c|}
\hline \multirow{6}{*}{ Coefficient $\beta_{0}$} & \multirow{3}{*}{ GDP } & \multicolumn{3}{|c|}{ The Fixed Effects Model (3.6) } & \multicolumn{3}{|c|}{ The Structural Equation Model (3.1)-(3.3) } \\
\hline & & \multirow{2}{*}{$\begin{array}{l}\text { Case } 1 \\
0.3032\end{array}$} & Case 2 & Case 3 & Case 1 & Case 2 & \multirow{2}{*}{$\frac{\text { Case : }}{0.796^{2}}$} \\
\hline & & & & 0.3501 & 0.8407 & & \\
\hline & & $(0.1232,0.4695)$ & & $(0.1664,0.5124)$ & $(0.7791,0.9655)$ & & $(0.7781,0.9249)$ \\
\hline & Price & & -0.0212 & -0.1078 & & -0.0707 & -0.1001 \\
\hline & & & $(-0.1784,0.1390)$ & $(-0.2106,-0.0046)$ & & $(-0.1134,-0.0225)$ & $(-0.1246,-0.0378)$ \\
\hline \multirow{9}{*}{$\begin{array}{l}\text { No. of Factors } \\
\text { Elasticity }\end{array}$} & $\operatorname{GDP}(-1)$ & 0.4151 & & 0.4334 & & & \\
\hline & & $(0.2387,0.6012)$ & & $(0.2585,0.6153)$ & & & \\
\hline & Price $(-1)$ & & 0.2150 & -0.0428 & & & \\
\hline & & & $(0.0512,0.3770)$ & $(-0.1481,0.0779)$ & & & \\
\hline & & & & & 1 & 1 & 1 \\
\hline & $E_{G}$ & 0.7184 & & 0.7835 & 0.7642 & & 0.7252 \\
\hline & & $(0.6704,0.7579)$ & & $(0.7397,0.8325)$ & $(0.7159,0.8449)$ & & $(0.7160,0.8151)$ \\
\hline & $E_{P}$ & & 0.1938 & -0.1505 & & -0.0621 & -0.0892 \\
\hline & & & $(0.1358,0.2608)$ & $(-0.1946,-0.1055)$ & & $(-0.1018,-0.0200)$ & $(-0.1031,-0.0486)$ \\
\hline
\end{tabular}


Table A.5: Results of the Constant Parameter Models from the Dataset of Liddle et al. (2020) (i.e., Dataset 4). The numbers in parentheses are the $95 \%$ confidence interval.

\begin{tabular}{|c|c|c|c|c|c|c|c|}
\hline \multirow{6}{*}{ Coefficient $\beta_{0}$} & \multirow{3}{*}{ GDP } & \multicolumn{3}{|c|}{ The Fixed Effects Model (3.6) } & \multicolumn{3}{|c|}{ The Structural Equation Model (3.1)-(3.3) } \\
\hline & & \multirow{2}{*}{$\begin{array}{l}\text { Case } 1 \\
0.7552\end{array}$} & \multirow[t]{2}{*}{ Case 2} & \multirow{2}{*}{$\begin{array}{l}\text { Case } 3 \\
0.7847\end{array}$} & \multirow{2}{*}{$\begin{array}{l}\text { Case } 1 \\
1.1590\end{array}$} & \multirow[t]{2}{*}{ Case 2} & \multirow{2}{*}{$\frac{\text { Case } 3}{0.9843}$} \\
\hline & & & & & & & \\
\hline & & $(0.4781,1.0755)$ & & $(0.4845,1.1437)$ & $(1.0057,1.3081)$ & & $(0.9634,1.1541)$ \\
\hline & Price & & 0.1080 & -0.0237 & & -0.0509 & -0.1136 \\
\hline & & & $(-0.0074,0.2240)$ & $(-0.1365,0.0618)$ & & $(-0.1131,0.0394)$ & $(-0.1510,-0.0072)$ \\
\hline \multirow{9}{*}{$\begin{array}{l}\text { No. of Factors } \\
\text { Elasticity }\end{array}$} & $\operatorname{GDP}(-1)$ & -0.1484 & & -0.0440 & & & \\
\hline & & $(-0.4626,0.1420)$ & & $(-0.3866,0.2780)$ & & & \\
\hline & Price $(-1)$ & & 0.0085 & -0.1003 & & & \\
\hline & & & $(-0.1121,0.1346)$ & $(-0.1908,0.0167)$ & & & \\
\hline & & & & & 1 & 1 & 1 \\
\hline & $E_{G}$ & 0.6068 & & 0.7407 & 1.1611 & & 0.9218 \\
\hline & & $(0.5228,0.6896)$ & & $(0.6520,0.8299)$ & $(1.0540,1.2544)$ & & $(0.9028,1.0356)$ \\
\hline & $E_{P}$ & & 0.1165 & -0.1239 & & -0.0376 & -0.1019 \\
\hline & & & $(0.0556,0.1784)$ & $(-0.1823,-0.0617)$ & & $(-0.0824,0.0301)$ & $(-0.1204,-0.0537)$ \\
\hline
\end{tabular}


Table A.6: Constancy Test

\begin{tabular}{lr}
\hline \hline & $p$-value \\
Case 1 & 0.0000 \\
Case 2 & 0.0000 \\
Case 3 & 0.0000 \\
\hline \hline
\end{tabular}

Figure A.1: Estimates of $\beta_{0}(\cdot)$ for Case 1 using the Data of All Countries (i.e., Dataset $1)$. Black, blue and red solid lines stand for the estimated $\beta_{0}(\cdot)$ using the bandwidths $h, h_{L}$ and $h_{R}$, respectively. The black dotted lines stand for the $95 \%$ confidence interval associated with the black solid line.

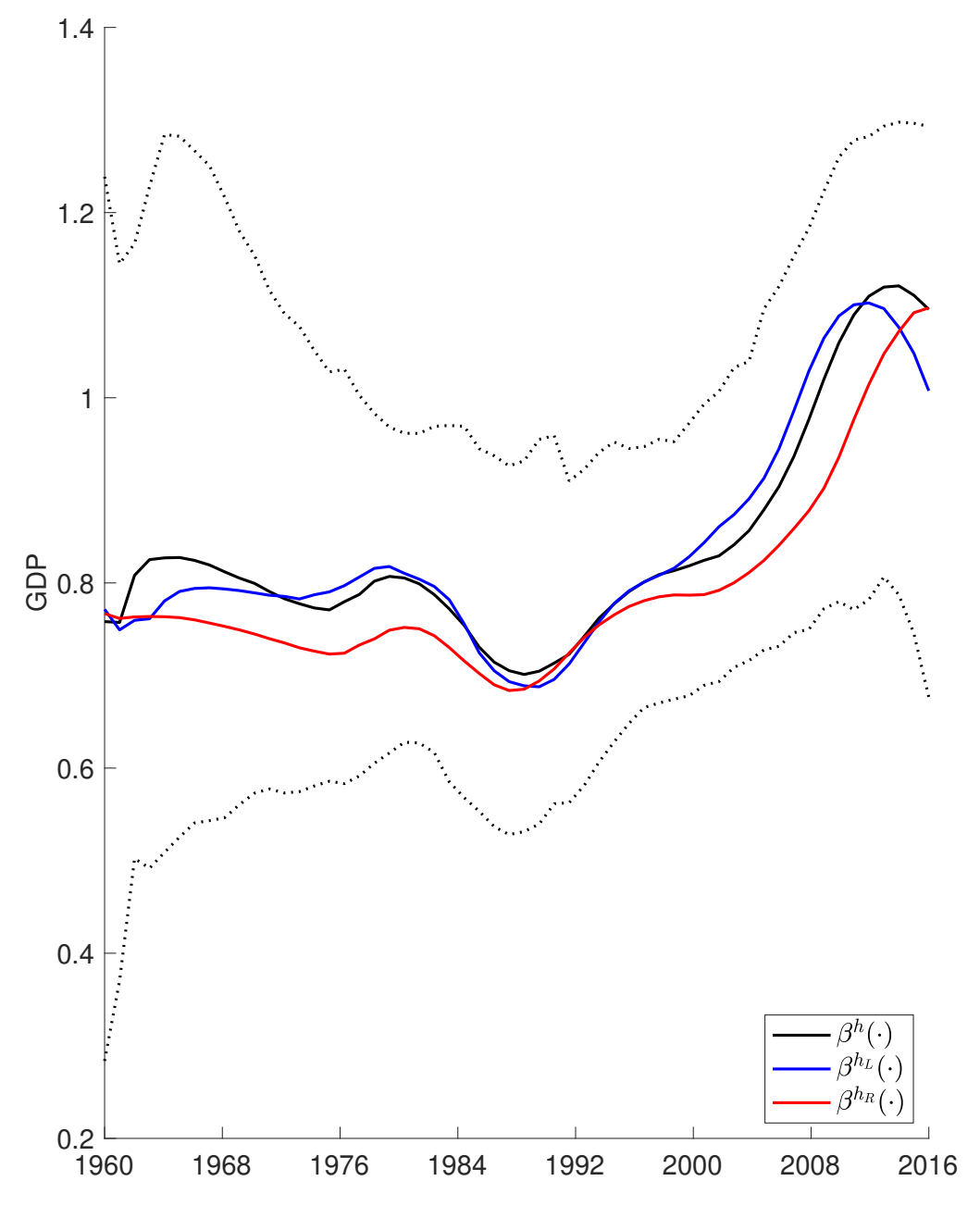


Figure A.2: Estimates of $\beta_{0}(\cdot)$ for Case 2 using the Data of All Countries (i.e., Dataset $1)$. Black, blue and red solid lines stand for the estimated $\beta_{0}(\cdot)$ using the bandwidths $h, h_{L}$ and $h_{R}$, respectively. The black dotted lines stand for the $95 \%$ confidence interval associated with the black solid line.

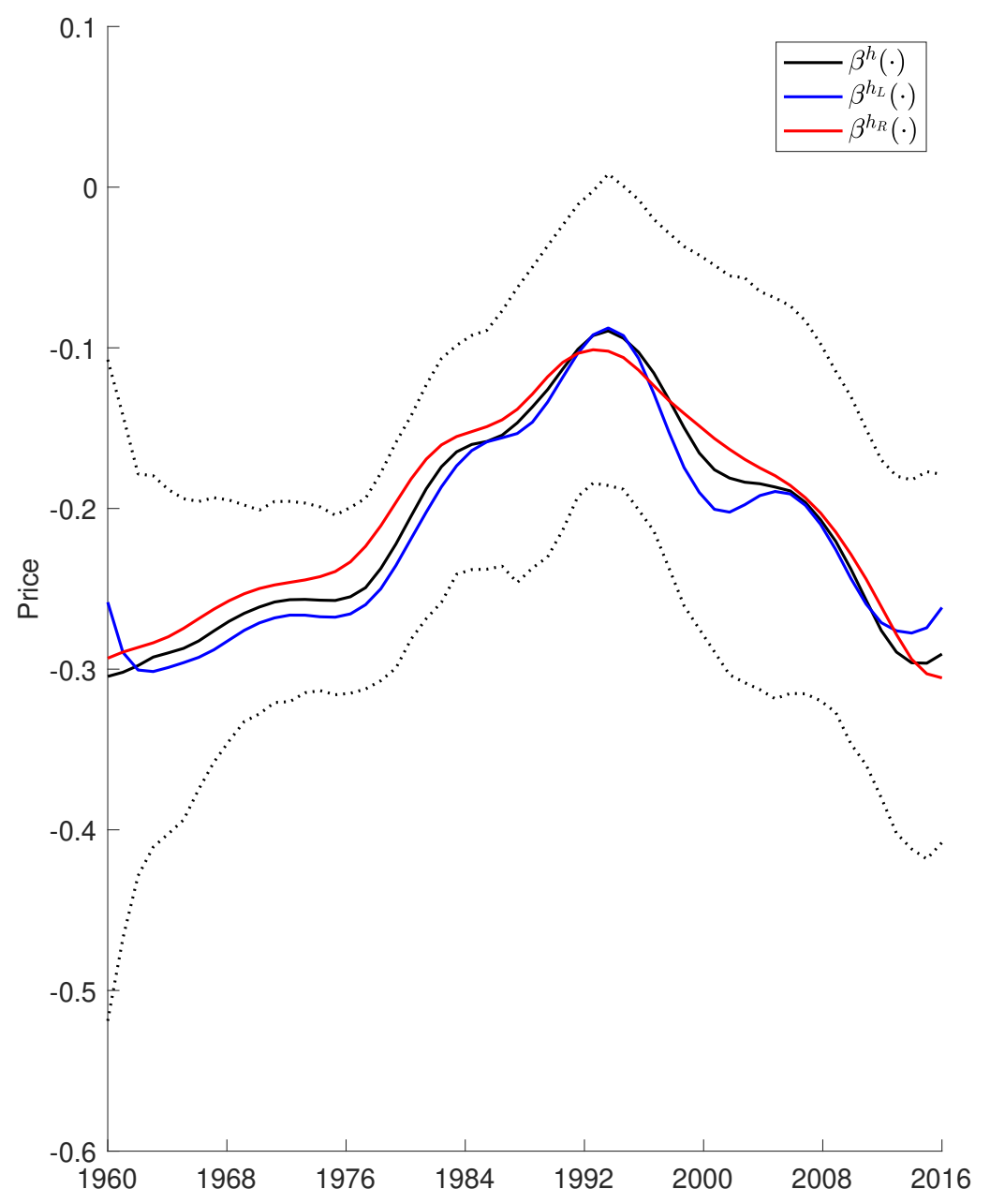


Figure A.3: Estimates of $\beta_{0}(\cdot)$ for Case 3 using the Data of All Countries (i.e., Dataset $1)$. Black, blue and red solid lines stand for the estimated $\beta_{0}(\cdot)$ using the bandwidths $h, h_{L}$ and $h_{R}$, respectively. The black dotted lines stand for the $95 \%$ confidence interval associated with the black solid line.
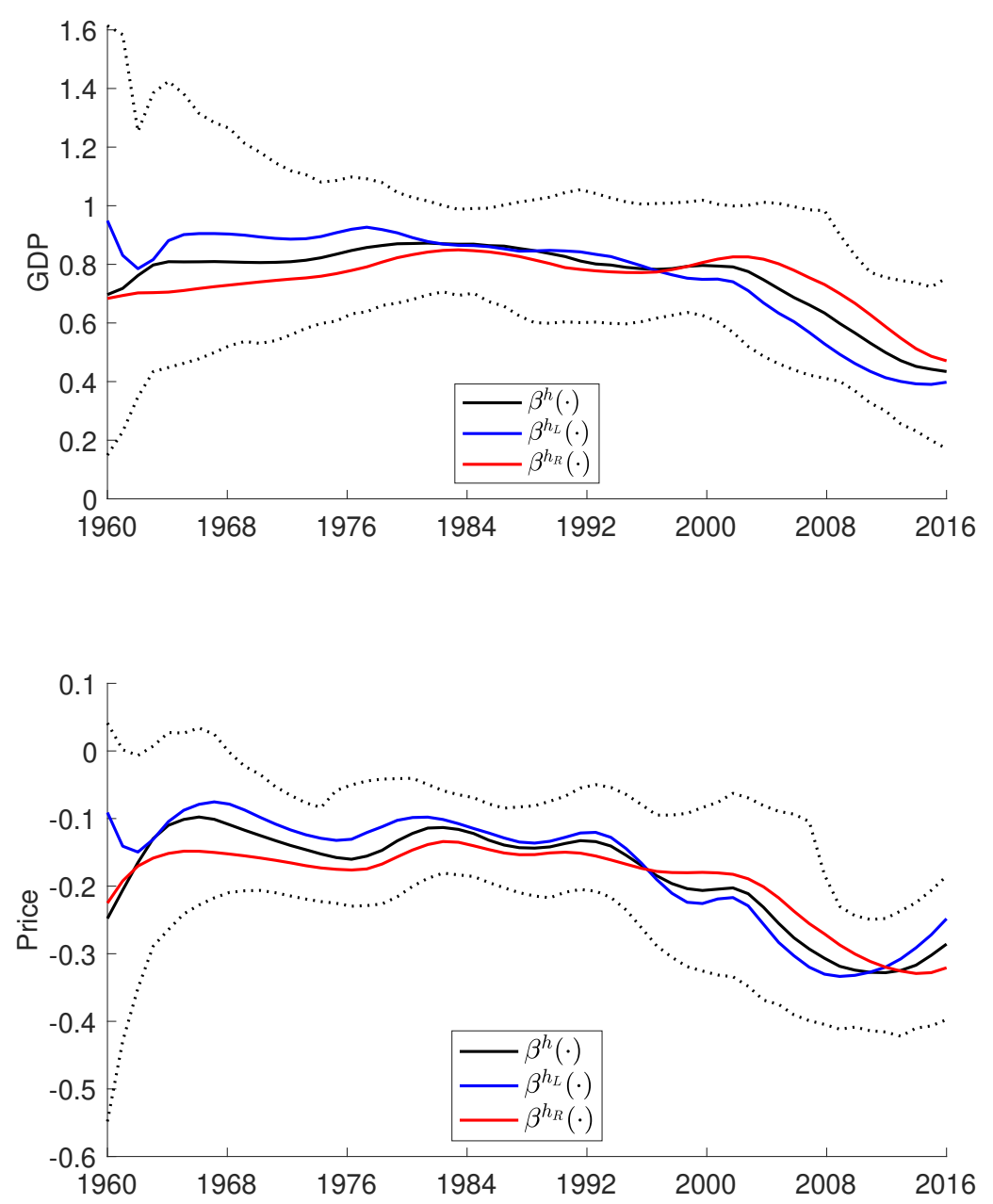
Figure A.4: Estimates of $\beta_{0}(\cdot)$ for Case 3 using the High Income Countries Data (i.e., Dataset 2). Black line stands for the estimated $\beta_{0}(\cdot)$. The black dotted lines stand for the $95 \%$ confidence interval.
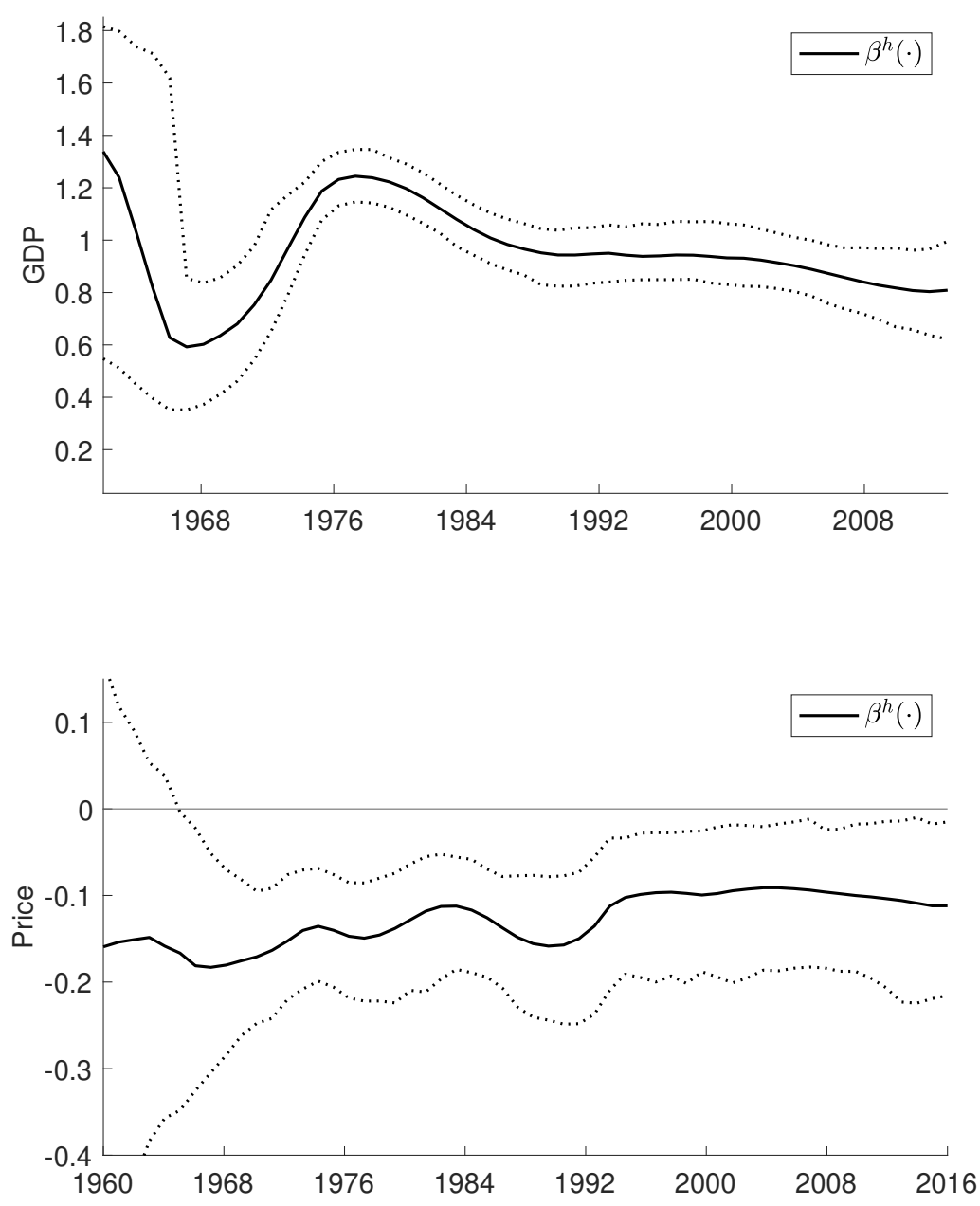
Figure A.5: Estimates of $\beta_{0}(\cdot)$ for Case 3 using the Middle Income Countries Data (i.e., Dataset 3). Black line stands for the estimated $\beta_{0}(\cdot)$. The black dotted lines stand for the $95 \%$ confidence interval.
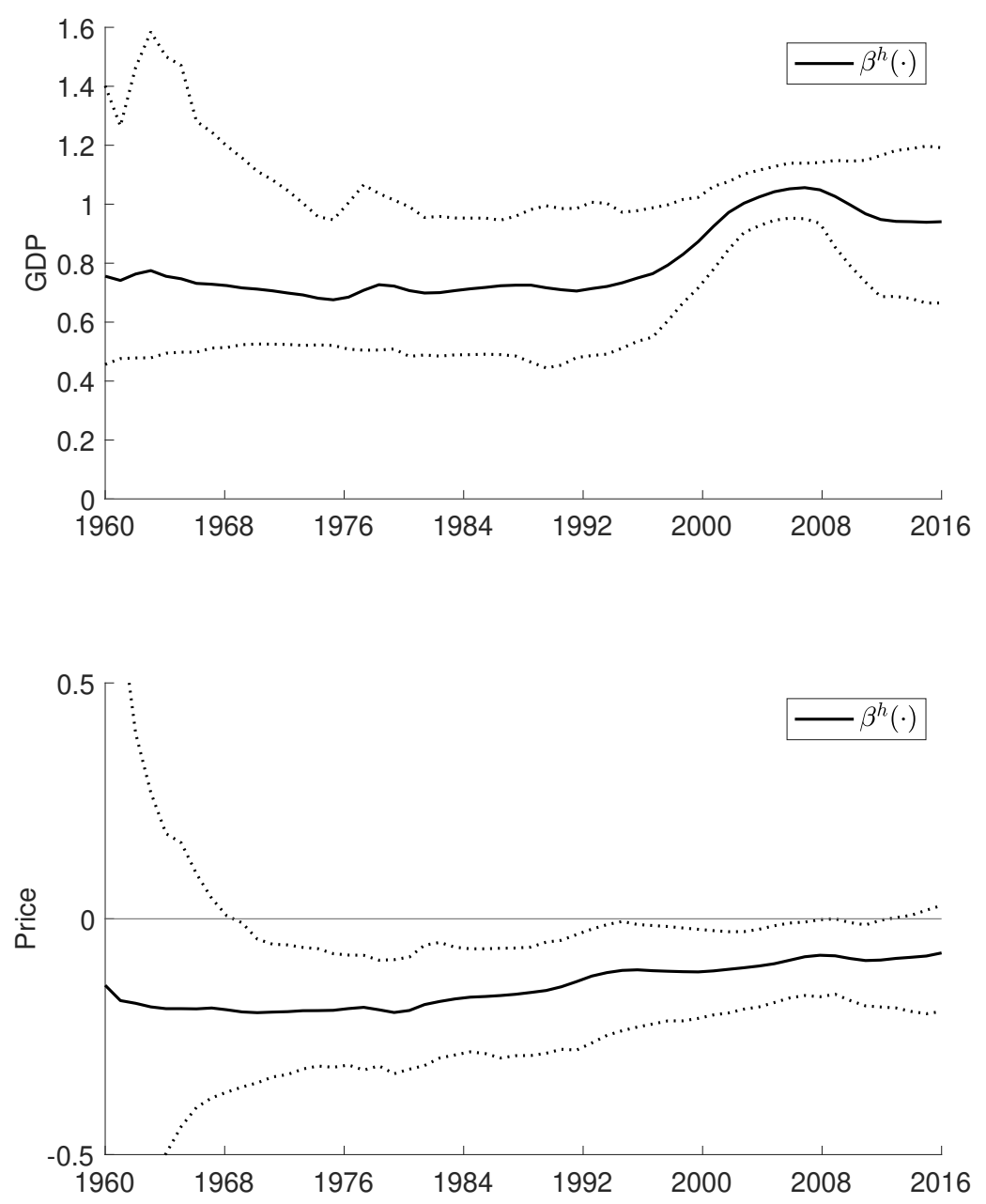
Figure A.6: Estimates of $\beta_{0}(\cdot)$ for Case 3 using the Data of Liddle et al. (2020) (i.e., Dataset 4). Black line stands for the estimated $\beta_{0}(\cdot)$. The black dotted lines stand for the $95 \%$ confidence interval.
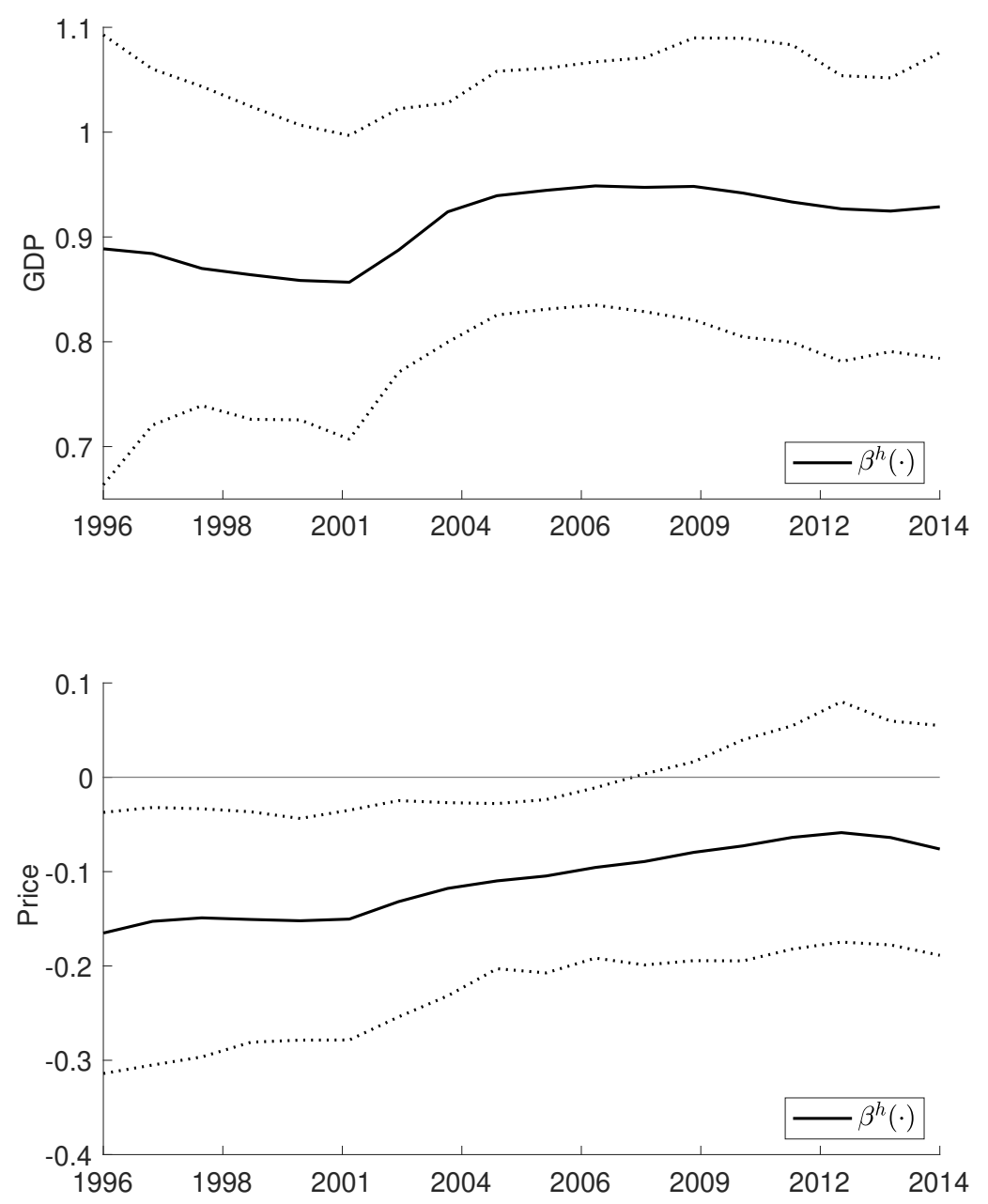
Figure A.7: Elasticity for Case 1 using the Data of All Countries (i.e., Dataset 1). Black, blue and red solid lines stand for the estimated elasticity using the bandwidths $h, h_{L}$ and $h_{R}$, respectively. The black dotted lines stand for the $95 \%$ confidence interval associated with the black solid line.

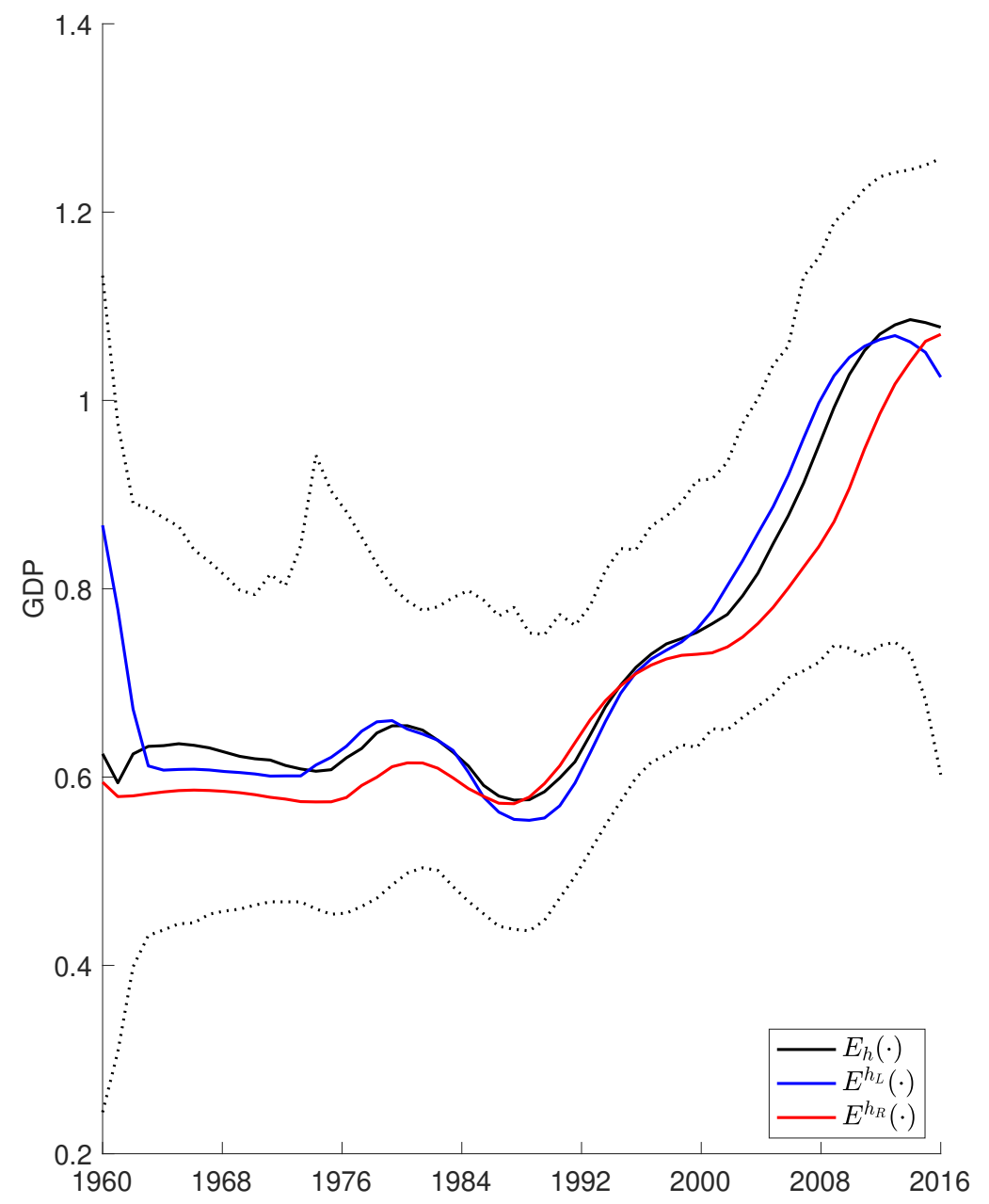


Figure A.8: Elasticity for Case 2 using the Data of All Countries (i.e., Dataset 1). Black, blue and red solid lines stand for the estimated elasticity using the bandwidths $h, h_{L}$ and $h_{R}$, respectively. The black dotted lines stand for the $95 \%$ confidence interval associated with the black solid line.

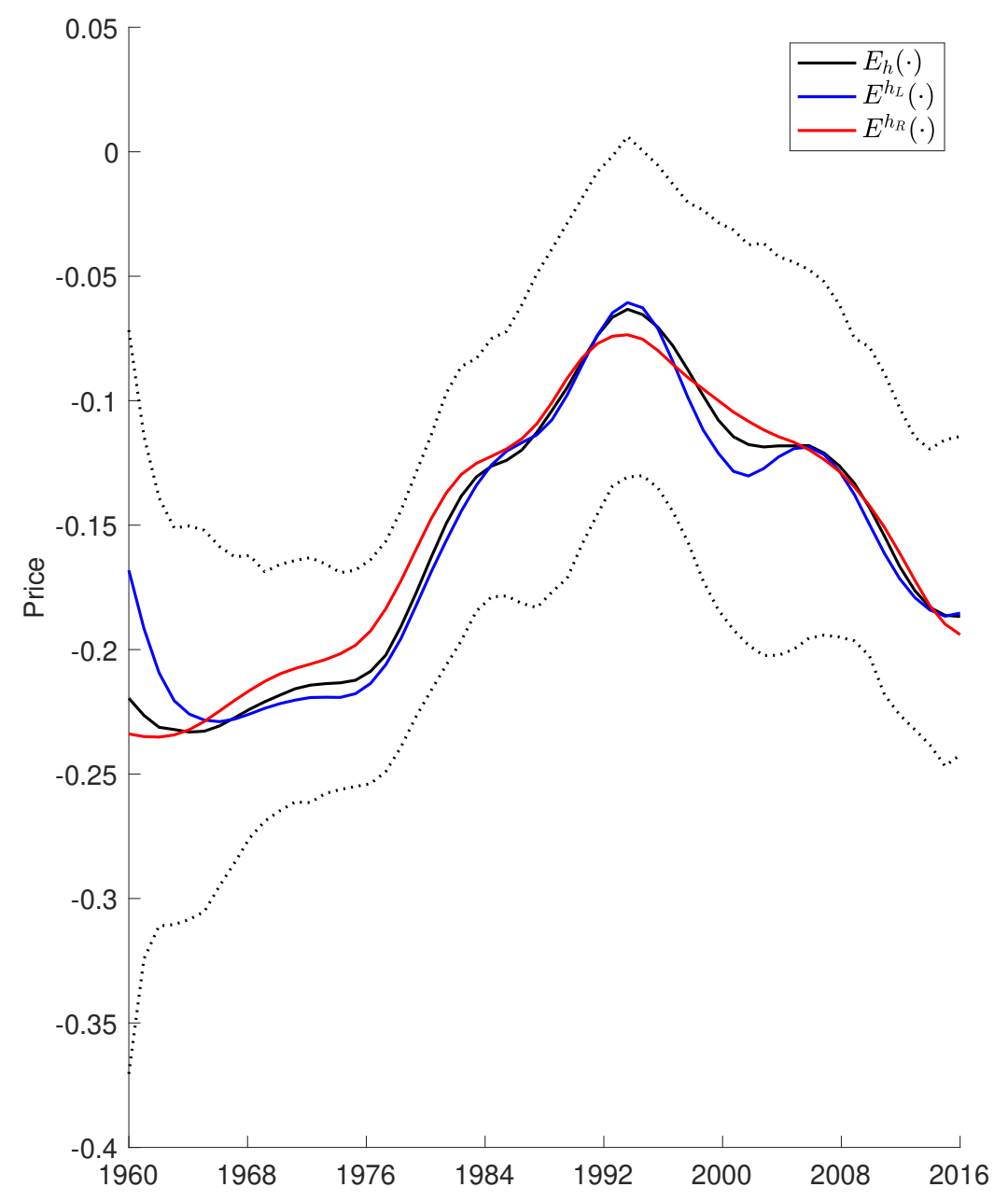


Figure A.9: Elasticity for Case 3 using the Data of All Countries (i.e., Dataset 1). Black, blue and red solid lines stand for the estimated elasticity using the bandwidths $h, h_{L}$ and $h_{R}$, respectively. The black dotted lines stand for the $95 \%$ confidence interval associated with the black solid line.
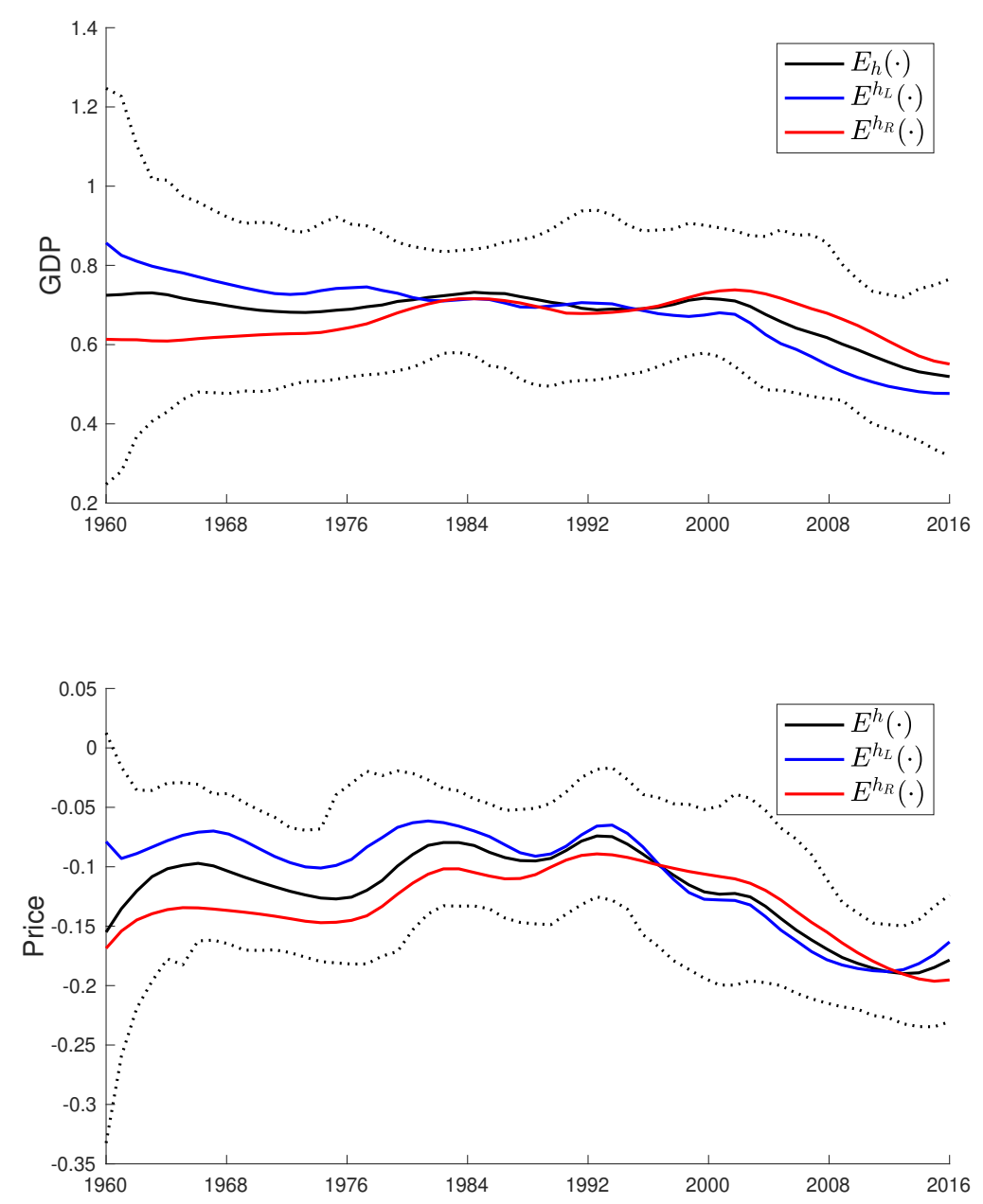
Figure A.10: Elasticity for Case 3 using the High Income Countries Data (i.e., Dataset 2 ). Black line stands for the estimated elasticity. The black dotted lines stand for the 95\% confidence interval.
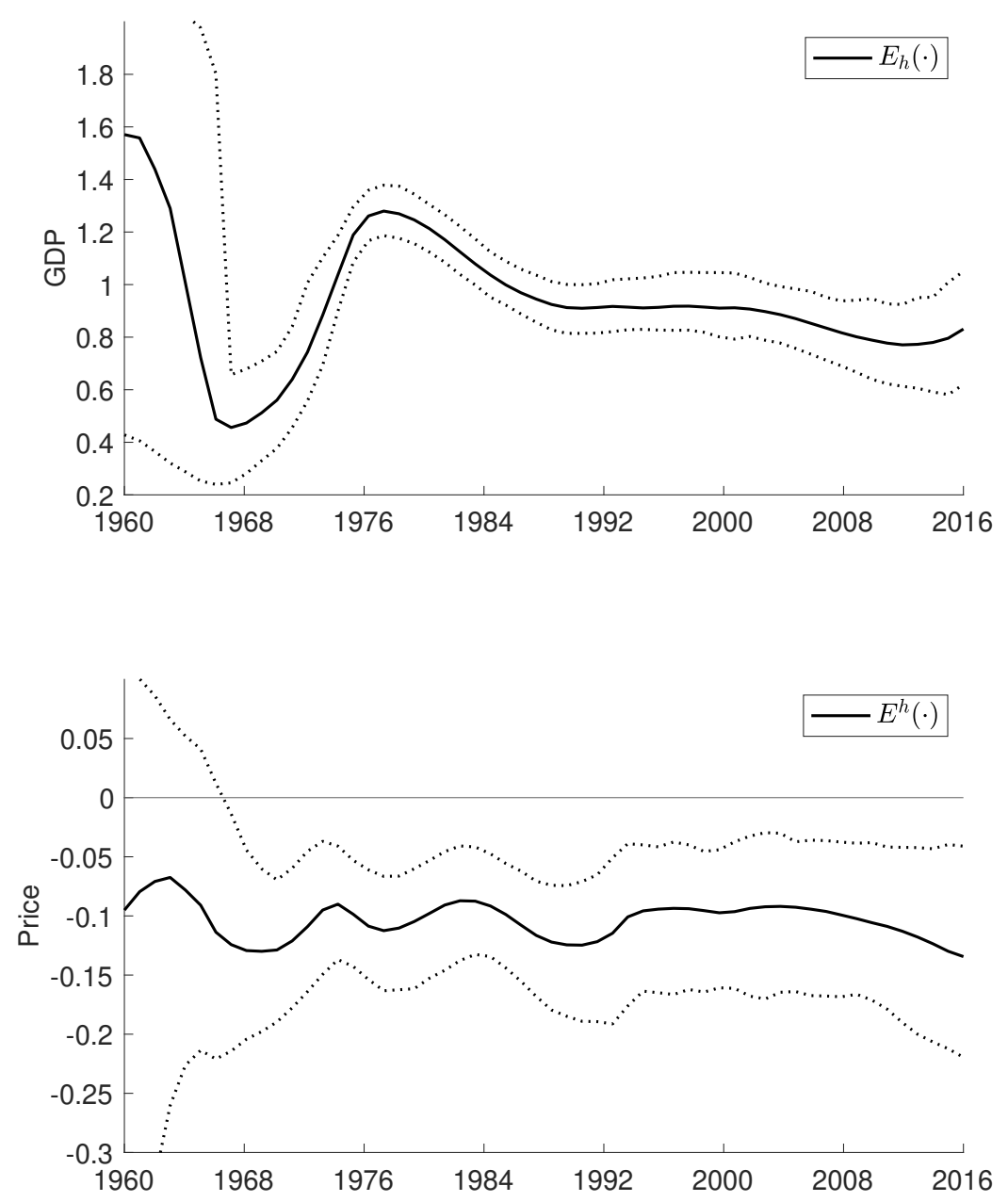
Figure A.11: Elasticity for Case 3 using the Middle Income Countries Data (i.e., Dataset 3). Black line stands for the estimated elasticity. The black dotted lines stand for the $95 \%$ confidence interval.
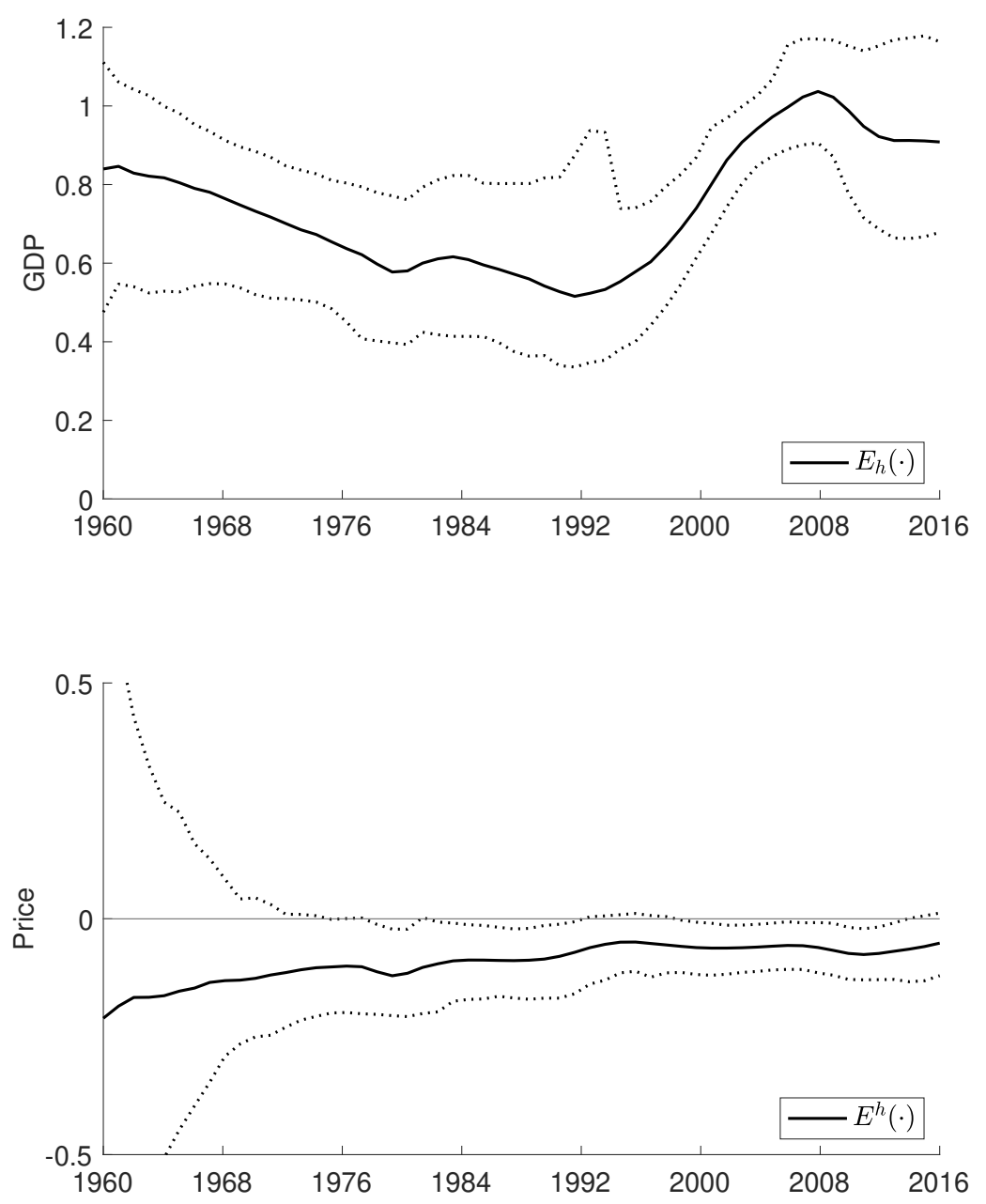
Figure A.12: Elasticity for Case 3 using the Data of Liddle et al. (2020) (i.e., Dataset 4). Black line stands for the estimated elasticity. The black dotted lines stand for the $95 \%$ confidence interval.
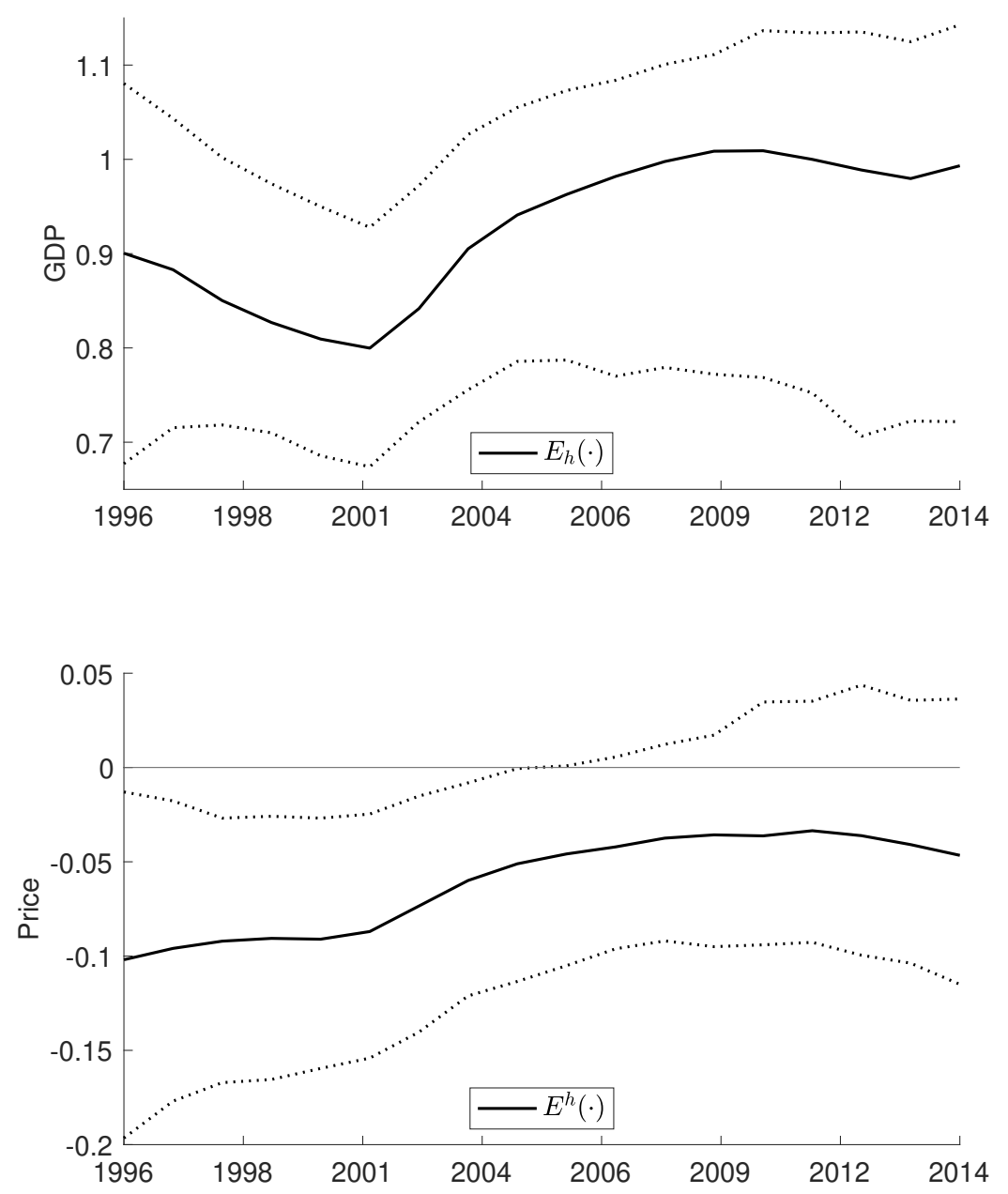